\title{
How should the lift and drag forces be calculated from 2-D airfoil data for dihedral or coned wind turbine blades?
}

\author{
Ang $\mathrm{Li}^{1}$, Mac Gaunaa ${ }^{1}$, Georg Raimund Pirrung ${ }^{1}$, Alexander Meyer Forsting ${ }^{1}$, and Sergio \\ González Horcas ${ }^{1}$ \\ ${ }^{1}$ Department of Wind Energy, Technical University of Denmark, Frederiksborgvej 399, DK-4000 Roskilde, Denmark \\ Correspondence: Ang Li (angl@dtu.dk)
}

\begin{abstract}
In the present work, a consistent method for calculating the lift and drag forces from the 2-D airfoil data for the dihedral or coned horizontal-axis wind turbines when using generalized lifting-line methods is described. The generalized lifting-line methods include, for example, lifting-line (LL), actuator line (AL), blade element momentum (BEM) and blade element vortex cylinder (BEVC) methods. A consistent interpretation of classic unsteady 2-D thin airfoil theory results for use in a generally moving frame of reference within a linearly varying onset velocity field reveals that it is necessary to use not only the relative flow magnitude and direction at one point along the chord line (for instance three-quarter-chord), but also the gradient of the flow direction in the chordwise direction (or, equivalently, the flow direction at the quarter-chord) to correctly determine the magnitude and direction of the resulting 2-D aerodynamic forces and moment. However, this aspect is generally overlooked and most implementations in generalized lifting-line methods use only the flow information at one calculation point per section for simplicity. This simplification will not change the performance prediction of planar rotors, but will cause an error when applied to non-planar rotors. The present work proposes a generalized method to correct the error introduced by this simplified single-point calculation method. In this work this effect is investigated using the special case, where the wind turbine blade has only dihedral and no sweep, operating at steady-state conditions with uniform inflow applied perpendicular to the rotor plane. We investigate the impact of the effect by comparing the predictions of the steady-state performance of non-planar rotors from the consistent approach with the simplified one-point approach of the LL method. The results are verified using blade geometry resolving Reynolds-averaged Navier-Stokes (RANS) simulations. The numerical investigations confirmed that the correction derived from thin airfoil theory is needed for the calculations to correctly determine the magnitude and direction of the sectional aerodynamic forces for non-planar rotors. The aerodynamic loads of upwind and downwind coned blades that are calculated using the LL method, the BEM method, the BEVC method and the AL method are compared for the simplified and the full method. Results using the full method, including different specific implementation schemes, are shown to agree significantly better with fully-resolved RANS than the often used simplified one-point approaches.
\end{abstract}

\section{Introduction}

With the scientific and engineering advancements in the design optimization and manufacturing of horizontal-axis wind turbines (HAWT), modern wind turbine blades are generally more flexible and may have more out-of-plane shapes compared to 
conventional stiff machines. Also, research on downwind turbines proposed for land-based low-rated-wind applications (Madsen et al., 2020b) and the ultra-light concept (Loth et al., 2012) involve wind turbine rotors with large out-of-plane shapes. Correctly predicting the aerodynamic loads on such non-planar rotors is important for the design optimization and verification of these new concepts. However, it is computationally expensive to use accurate blade geometry resolving Navier-Stokes simulations for aerodynamic calculations, especially during the design optimization phase. Instead, low- to mid-fidelity generalized lifting-line aerodynamic models that use 2-D airfoil data provided by Navier-Stokes solvers or wind tunnel measurements are widely used. The models range from the low-fidelity blade element momentum (BEM) method (Madsen et al., 2020a) and blade element vortex cylinder (BEVC) method (Li et al., 2021) to the higher-fidelity lifting-line (LL) method (Phillips and Snyder, 2000) and actuator line (AL) method (Sørensen and Shen, 2002). The advantage of such models is that it is not necessary to directly solve the Navier-Stokes equations for the whole flow domain and resolve the 3-D blade geometry. In addition, the so-called engineering dynamic stall model is usually applied to such generalized lifting-line methods to account for unsteady 2-D effects. However, the dynamic stall name of the model is misleading because the model is also active for nonstalled conditions. Therefore a more proper name for such models is unsteady 2-D airfoil aerodynamic models. For example, in the Beddoes-Leishman type dynamic stall model (Hansen et al., 2004; Pirrung and Gaunaa, 2018) that is implemented in the HAWC2 code (Larsen and Hansen, 2007; Madsen et al., 2020a), the following effects are modeled: unsteady attached flow, unsteady flow separation (dynamic stall), non-circulatory lift force and the effective pitch rate drag force.

According to unsteady 2-D thin airfoil aerodynamics, it is necessary to use the flow information at both the quarter-chord point and the three-quarter-chord point to correctly determine the magnitude and direction of the lift and drag forces (Bergami and Gaunaa, 2012; Pirrung and Gaunaa, 2018). However, this aspect is generally overlooked and is often not a focus of engineering wind turbine aerodynamic model descriptions (Schepers et al., 2021). For simplicity, most implementations only use the flow information at one calculation point per section. This simplification will not change the performance prediction of planar rotors, but will cause an error for non-planar rotors. In this work, we focus on a special case where the blade has only dihedral and no sweep, operating at steady-state conditions with uniform inflow applied perpendicular to the rotor plane. We investigate the impact of the one-point simplification by comparing the results from the LL method using a two-point approach against results following the one-point simplification for non-planar rotors. Furthermore, the results computed from blade geometry resolving Reynolds-averaged Navier-Stokes (RANS) simulations are used for comparison. The numerical investigations showed that correctly determining the magnitude and direction of the lift force are important for non-planar rotors. We test the corrections to the simplified one-point approaches thoroughly. The aerodynamic loads of upwind and downwind coned blades are calculated using the LL method, the AL method, the BEM method and the BEVC method, both without and with the correction. In addition, the analytical derivations and numerical results in this work show that the total non-circulatory force is negligible for such steady-state conditions.

The structure of the present work is as follows: the highlights from the unsteady 2-D thin airfoil theory are firstly summarized in Sect. 2. This section also includes the interpretation of these results in terms of what is necessary to be included in generalized lifting-line methods. Then, the non-circulatory forces of a pure dihedral blade under steady-state operating conditions are derived in Sect. 3. Subsequently, the corrections for the generalized lifting-line method that only uses one chordwise calculation 
point are derived in Sect. 4. Then, different aerodynamic models for the comparison are described in Sect. 6. In Sect. 7, the setup of the numerical tests is described and the results from different generalized lifting-line methods are compared together with the results from the blade geometry resolving RANS solver-both without and with the corrections. Finally, the conclusions and the future work are summarized in Sect. 8.

\section{Highlights from unsteady 2-D thin airfoil aerodynamics}

Unsteady 2-D thin airfoil theory is of vital importance to correctly model the aerodynamics of wind turbine blades with dihedral using the generalized lifting-line methods. The reason is related to the ideas underlying generalized lifting-line methods, which are considered as the application of perturbation theory (Van Dyke, 1975). The aerodynamics of the full 3-D system is considered as the combination of an inner and an outer problem. The inner problem is the unsteady 2-D airfoil aerodynamics that has simple geometry (2-D) but locally complex flow around the airfoil section (Navier-Stokes equations). The outer problem is the 3-D rotor and wake problem that has complex geometry but irrotational flow. The full 3-D aerodynamic problem is approximately solved by solving the two problems together (Johnson, 2013). Analysis using unsteady 2-D thin airfoil theory reveals that this problem is driven by the relative velocities that would have been there if the velocities induced by the 2-D airfoil and its wake were not present. To find this situation for the inner problem, the 3-D flow, including the 3-D induction minus the local 2-D induction, the onset flow and the airfoil motion, at each blade section is transformed into the 2-D airfoil section coordinate system, where it is used to solve the inner problem. The results from the inner 2-D problem, which are the sectional forces and the bound circulation, are used as the input for solving the 3-D problem.

In this section, some of the important conclusions and results from unsteady 2-D thin airfoil theory are briefly described. These conclusions will be used to derive the non-circulatory forces in Sect. 3 and to determine the magnitude and direction of the lift force in Sect. 4. A detailed derivation of 2-D unsteady thin airfoil theory, also including the analysis of details behind a consistent evaluation of unsteady drag, is shown in Gaunaa (2010). This previous work also considers the effect of a deformable/morphable camber line. But if the camber line shape is not changing over time, the classical steady and unsteady 2-D thin airfoil results are recovered. It is seen from the results of the analysis that the local pressure difference anywhere on the thin airfoil is determined from the undisturbed relative local flow velocities and their time history over the whole airfoil chord length. It doesn't matter how the relative flow velocities (and their time history) arise; if two situations have the same "input", the resulting pressure differences over the airfoil will be the same. This information is crucial for the interpretation of the thin airfoil results in the more general case, where the thin airfoil framework results are to be used "inside" the framework of the general lifting-line methods. More specifically, it is the magnitude of the relative wind speed and the local normal flow velocity (the component of the relative flow velocity in the direction locally normal to the chord line) that drive the solution of the local pressure differences, and thereby also the integral airfoil section forces.

For the present work, we consider the simplest representation of an uncambered airfoil, a flat plate, with the chord length of $c=2 b$ which is placed in a uniform flow with the free stream velocity of $V$. The blade motion is described by a heaving motion $\dot{y}$ (positive upward, perpendicular to the incoming flow), a pitching motion $\dot{\theta}$ about the axis at $\xi=a b$ (positive nose-up) and 
a horizontal motion of $\dot{x}$ (positive direction downwind). A sketch of the coordinate system and the geometry of a flat plate to derive the unsteady 2-D thin airfoil theory is shown in Fig. 1.

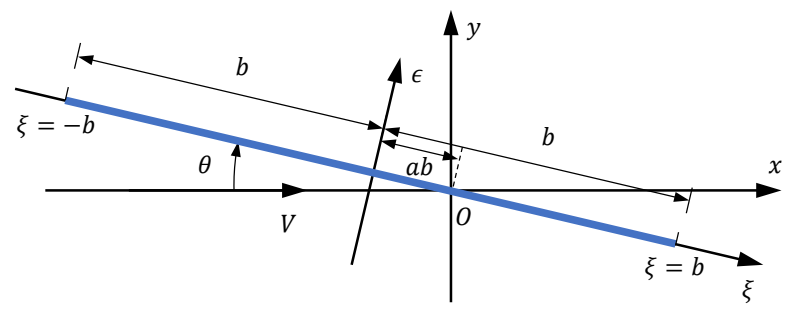

Figure 1. Definitions of the coordinate system and positive directions used in the derivations of the unsteady 2-D thin airfoil theory.

\section{5}

\subsection{The lift force magnitude}

Essentially, the fundamental output from the thin airfoil analysis is the local unsteady pressure difference over the airfoil and the leading edge suction force. At the leading edge of an airfoil, there is generally a low pressure because the air is accelerated around the relatively small leading edge radius. In thin airfoil theory the airfoil thickness, and along with it the leading edge radius, tend to zero. This results in the pressure tending to minus infinity at the leading edge ${ }^{1}$, which has an effective area tending to zero. The leading edge suction force can be considered the limit of the pressure acting on the leading edge area as the leading edge radius tends to zero. This provides a force in the airfoil tangential direction, pointing from the trailing edge to the leading edge. The pressure difference over the airfoil in the flat airfoil case acts locally normal to the airfoil surface, and therefore in this flat-airfoil case only in the normal $(\epsilon)$ direction. Therefore the integral forces on the airfoil consist of the contributions from the normal force $N$ (in the $\epsilon$ direction) and the tangential force $T$ (in the direction opposite to $\xi$ ). The force components usually used in generalized lifting-line methods are lift and drag, which are defined relative to the local flow direction and not relative to the orientation of the airfoil.

$L=N \cos \left(\theta+\Delta \alpha_{r}\right)-T \sin \left(\theta+\Delta \alpha_{r}\right)$

In the equation above, $\Delta \alpha_{r}$ is the contribution from the airfoil motion to the angle of attack. In the derivation of thin airfoil theory, it is assumed that the angles of attack are small and that the relative velocities due to airfoil motion are significantly smaller than the onset flow velocity. Under these assumptions the detailed definition of the angle of attack is not important for the evaluation of the lift force because $\left|\theta+\Delta \alpha_{r}\right| \ll 1$ and $|T| \ll|N|$, which inserted into Eq. (1) leads to

$L \approx N$

\footnotetext{
${ }^{1}$ This correspond to the singularity of the resulting bound vorticity distributions at the leading edge of the thin airfoil.
} 
Using the expression for $N$ derived in (Gaunaa, 2010) we get the lift force for the oscillatory case ${ }^{2}$

$L=\underbrace{2 \pi \rho b(V-\dot{x})\left[(V-\dot{x}) \theta-\dot{y}+\left(\frac{1}{2}-a\right) b \dot{\theta}\right] C(k)}_{L^{C}=L^{Q S} C(k)}+\underbrace{\pi \rho b^{2}[(V-\dot{x}) \dot{\theta}-\ddot{x} \theta-\ddot{y}-a b \ddot{\theta}]}_{L^{N C}}$

where $C(k)$ is Theodorsen's lift deficiency function (Theodorsen, 1935) and $k$ is the reduced frequency

$k=\Omega b / V$

The first term of the lift force is the circulatory lift force, $L^{C}$, which through Theodorsen's lift deficiency function takes the unsteady 2-D shed wake into account if temporal variations in the quasi-steady lift, $L^{Q S}$, is present. If $L^{Q S}$ is constant, then $C(k)=1$. The second lift term, $L^{N C}$, is the non-circulatory lift force, which always acts instantaneously without any time-lag.

\subsubsection{Circulatory lift}

The circulatory lift $L^{C}$ is determined by the quasi-steady lift $L^{Q S}$ and the lift deficiency function $C(k)$ as shown in Eq. (3). To have a better understanding of the circulatory lift equation in the context of the generalized lifting-line methods, it is convenient to express it as an explicit function of the angle of attack. To determine the angle of attack more precisely, we need first to consider how it is defined. The component of the relative flow velocity in the direction perpendicular to the airfoil ( $\epsilon$-direction) is usually termed the up-wash. The up-wash at a chordwise position with the coordinate of $\xi$ is:

$w=(V-\dot{x}) \theta-\dot{y}+(\xi-a b) \dot{\theta}$

By setting $\xi=\frac{1}{2} b$, we obtain the up-wash at the three-quarter-chord point.

$w_{3 / 4}=(V-\dot{x}) \theta-\dot{y}+\left(\frac{1}{2}-a\right) b \dot{\theta}$

Since an angle of attack is defined as the angle between a flow direction and the chord line, we have in this case: ${ }^{3} \alpha_{3 / 4}=$ $w_{3 / 4} /(V-\dot{x})$, such that the circulatory lift in Eq. (3) can be written as:

$L^{C}=2 \pi \rho b(V-\dot{x}) w_{3 / 4} C(k)=\frac{1}{2} \rho(V-\dot{x})^{2} c 2 \pi \alpha_{3 / 4} C(k)$

This means the magnitude of the quasi-steady lift is correctly determined by using the angle of attack at the three-quarterchord point. In thin airfoil theory, $C_{L}^{Q S}=2 \pi \alpha_{3 / 4}$. After furthermore noting that under the thin airfoil approximations, the relative wind speed is equal to $(V-\dot{x})$, we can finally write the circulatory lift expression in terms of the 2-D lift airfoil data as:

$L^{C}=L^{Q S} C(k)$

where

$L^{Q S}=\frac{1}{2} \rho V_{\mathrm{rel}}^{2} c C_{L}^{Q S}\left(\alpha_{3 / 4}\right)$

\footnotetext{
${ }^{2}$ In the case where the forcing is arbitrary, the 2-D shed wake effect can be modeled by a time-lag filter on the term $(V-\dot{x}) \theta-\dot{y}+\left(\frac{1}{2}-a\right) b \dot{\theta}$, as shown in Gaunaa (2010).

${ }^{3}$ Under the usual thin airfoil small angle approximation $\tan \alpha_{3 / 4} \approx \alpha_{3 / 4}$.
} 


\subsubsection{Non-circulatory lift}

The non-circulatory lift in Eq. (3) is summarized as follows.

$L^{N C}=\pi \rho b^{2}[(V-\dot{x}) \dot{\theta}-\ddot{x} \theta-\ddot{y}-a b \ddot{\theta}]$

According to Eq. (10), the pitch rate of the airfoil $\dot{\theta}$, the pitching acceleration of the airfoil $\ddot{\theta}$, the heave acceleration of the airfoil $\ddot{y}$ as well as the streamwise acceleration of the airfoil $\ddot{x}$ will all contribute to the non-circulatory lift.

\subsection{A consistent method for determining 2-D drag}

One of the key conclusions that can be drawn from a full unsteady 2-D thin airfoil theory analysis regarding application in generalized lifting-line methods is usually overlooked: a consistent definition of the direction with which lift and drag forces are defined. Even though the details may at first glance seem overwhelmingly focused on unimportant details, it will be shown at the end of this section that the effect of skipping these details can in some cases lead to completely unphysical behaviors.

As stated previously, the local angle of attack observed at different locations on the chord line differs from each other in the general unsteady case due to the pitching/torsional motion of the blade. By use of Eq. (5), the angle of attack observed in the chordwise position $\xi$ can be determined.

$\alpha(\xi)=\frac{w(\xi)}{(V-\dot{x})}=\frac{(V-\dot{x}) \theta-\dot{y}+(\xi-a b) \dot{\theta}}{(V-\dot{x})}$

The result of the analysis will reveal that there is a special significance of the quarter-chord point, $\xi=-b / 2$, so we skip the complexity of performing a more general analysis and consider directly the angle of attack at the quarter-chord point.

$\alpha_{1 / 4}=\frac{w\left(\xi=-\frac{1}{2} b\right)}{(V-\dot{x})}=\frac{(V-\dot{x}) \theta-\dot{y}-\left(\frac{1}{2}+a\right) b \dot{\theta}}{(V-\dot{x})}=\alpha_{3 / 4}-\frac{b \dot{\theta}}{(V-\dot{x})}$

Using this angle of attack as the reference with which the drag force is defined, we apply the usual thin airfoil small angle approximation.

$D_{1 / 4}=N \sin \alpha_{1 / 4}-T \cos \alpha_{1 / 4} \approx N \alpha_{1 / 4}-T$

The tangential force, which in the uncambered airfoil case stems entirely from the leading edge suction force, is for the flat airfoil case given by Gaunaa (2010) as:

$T=\frac{\pi}{2} \rho b\left(2(V-\dot{x}) \alpha_{3 / 4} C(k)-b \dot{\theta}\right)^{2}$

Inserting the results from Eqs. (2), (3), (12) and (14) into Eq. (13), it can be shown that:

$D_{1 / 4}=-\underbrace{L^{C} \Delta \alpha_{i}}_{D_{i}}+\underbrace{L^{N C} \alpha_{1 / 4}}_{D^{N C}}-\underbrace{\frac{1}{2} \pi \rho b^{3} \dot{\theta}^{2}}_{D_{\text {pitchrate }}}$ 
where $\Delta \alpha_{i}$ can be considered as the effective induced angle of attack due to the unsteady wake, which by use of $V_{\text {rel }}=(V-\dot{x})$ can be written as

$\Delta \alpha_{i}=\frac{L^{C}-L^{Q S}}{2 \pi \rho b V_{\mathrm{rel}}^{2}}$

Each component of the drag force in Eq. (15) will be described briefly below.

\subsubsection{Shed wake induced drag}

The term $D_{i}$ in Eq. (15) is the induced drag force, which is an effective drag that originates from the changed direction of the circulatory lift force due to the induced velocity from the shed vorticity in the wake after an unsteady airfoil. For steady-state conditions, the bound circulation strength does not change with time, which means that there is no shed-wake induced drag force. This is in agreement with the term in Eq. (16), where for steady conditions $L^{C}=L^{Q C}$, which results in zero induced unsteady 2-D drag, $D_{i}=0$.

\subsubsection{Non-circulatory drag}

In Eq. (15), the drag component of $D^{N C}$ is the effective drag force due to the projection of the non-circulatory lift $L^{N C}$. It is seen that the direction of the non-circulatory force is perpendicular to the chord.

\subsubsection{Drag due to pitch rate}

The last term $D_{\text {pitchrate }}$ of the drag force is due to the pitch rate, and is in some sense a drag-equivalent to the non-circulatory lift term due to the pitch rate, $\pi \rho b^{2}(V-\dot{x}) \dot{\theta}$. When the airfoil has a pitching motion with the pitch rate $\dot{\theta}$, there will be a linear variation of up-wash along the chord, which results in this drag term. The magnitude of this drag component is proportional to the square of the pitch rate $\dot{\theta}$. For the airfoils with low to moderate reduced frequency, this term is negligible.

\subsubsection{Using a different reference direction for drag}

In analogy with Eq. (13), the drag defined from the angle of attack in the three-quarter-chord point turns out to be:

$D_{3 / 4}=N \sin \alpha_{3 / 4}-T \cos \alpha_{3 / 4} \approx N \alpha_{3 / 4}-T=D_{1 / 4}+L \frac{\dot{b}}{(V-\dot{x})}$

For cases with non-negligible pitch rate, the differences in the two different drag values, Eqs. (15) and (17), can be quite substantial. In some cases, it is even possible that the contribution from the last term in the three-quarter-chord-reference based drag can have a steady negative value. Note that both situations reflect the same physics and also exactly the same total force magnitude and direction. As long as the reference directions are handled correctly both methods give exactly the same correct result.

In order to shed light on the aspect we consider here, the extreme case where ideal 2-D thin airfoil sections are mounted on a spinning vertical axis wind turbine (VAWT) operating with zero onset velocity is investigated. The airfoils are set at an 
angle such that a constant outward lift is generated due to the relative wind speed from the rotation of the VAWT. We note that $(V-\dot{x})=V_{\text {rel }}=\Omega R$, where $\Omega$ and $R$ are the angular speed and radius of the VAWT, respectively. In this case, the flow situation as observed from the airfoil section does not change over time, causing the induced drag term to vanish. In addition, there is no streamwise acceleration $\ddot{x}$ or pitching acceleration $\ddot{\theta}$ of the airfoil. The steady situation does have a constant and nonzero pitch rate and heave acceleration $\dot{\theta}=-\Omega$ and $\ddot{y}=-\Omega^{2} R$. When evaluating the terms in the non-circulatory lift in Eq. (3), it is seen that the pitch rate term exactly cancel out the heave acceleration term, such that the total lift contains only the circulatory part. Due to the steady nature of the setup the circulatory lift is equal to the quasi-steady lift, which in turn is freely adjustable by setting the constant angle of the airfoil on the VAWT. Turning now to the drag, we see that all terms in the quarter-chord-reference drag Eq. (15) vanish, resulting in a thin airfoil quarter-chord drag of exactly zero. This is in agreement with the steady-state 2-D thin airfoil drag of zero following d'Alembert's paradox. If we evaluate instead the three-quarterchord-reference drag value we get from Eq. (17)

$D_{3 / 4, \mathrm{VAWT}}=L \frac{b \dot{\theta}}{(V-\dot{x})}=-L \frac{b \Omega}{\Omega R}=-L \frac{c}{2 R}$

In non-dimensional coefficients this corresponds to a 2-D drag coefficient of $C_{D, 3 / 4, \mathrm{VAWT}}=-C_{L} c /(2 R)$. As mentioned above, we can freely adjust the mounting angle of the airfoil on the spinning VAWT to obtain lift coefficients of either positive or negative signs that we desire. This also means that in this case the correct value of the three-quarter-chord-reference drag may even be a constant negative value. As stated previously, this situation describes the same physics as well as the same force magnitude and direction no matter what reference is used to define the drag force. It is the change in reference direction that makes the three-quarter-chord-reference drag value seem counterintuitive. For this reason, it is suggested to use the quarter-chord-reference direction definition of the drag coefficient in aeroelastic codes. Figure 2 shows schematically the two implementations described previously.

As the last point in this section it should be mentioned, that the effect of applying the erroneous definition of the direction of the forces in the VAWT case will lead to an effective drag error of the magnitude. For example, for the case of using the three-quarter-chord reference direction but using $D_{1 / 4}$ instead of $D_{3 / 4}$, there will be an effective drag error as given by Eq. (18). This can be illustrated in Fig. 2 (b) that the erroneous total force will be $L$ instead of $F_{t o t}$. For this reason an erroneously implemented model may show that it is possible for a VAWT to produce positive aerodynamic power even without an onset flow, as also observed by Pirrung and Gaunaa (2018). These important details about the drag are generally overlooked but are important for the performance prediction of dihedral blades, which will be investigated in Sect. 4. It should be emphasized that the considerations in this section are derived from the thin airfoil theory, which does not include the effects of steady 2-D profile drag. This extra drag component from the 2-D airfoil polars has to be added to the contributions from the thin airfoil theory in the generalized lifting-line methods. 


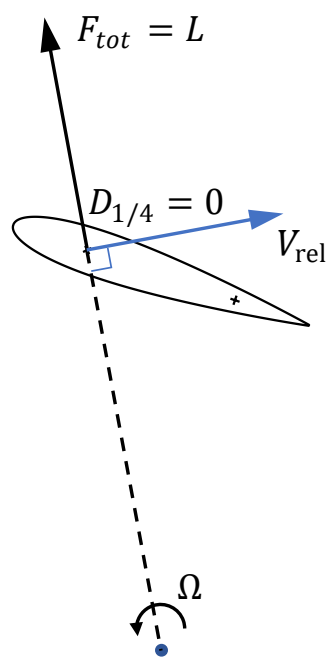

(a)

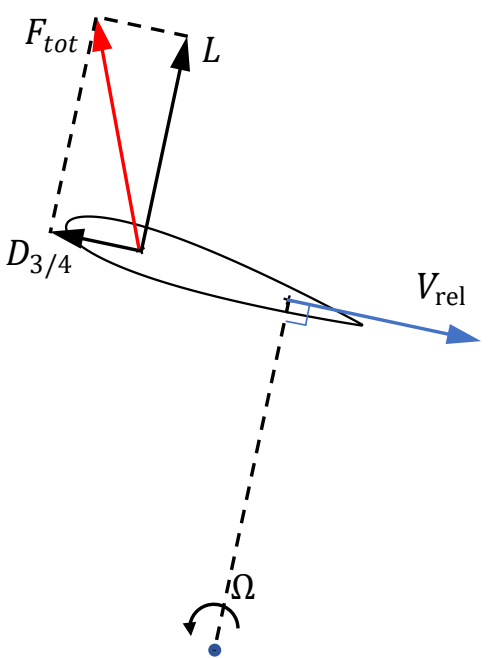

(b)

Figure 2. Illustration of two correct methods of defining the direction of the lift and drag forces for a VAWT. (a) Correctly using $D_{1 / 4}$ and relative direction at the quarter-chord point. (b) Correctly using $D_{3 / 4}(<0)$ and relative direction at the three-quarter-chord point. The total force vector in the two cases is identical.

\section{Non-circulatory lift of a pure dihedral blade}

In this section, we focus on the special case that the rotor is non-planar and the blades have no sweep. The blades are operating at steady-state, with uniform inflow applied perpendicular to the rotor plane and the rotor has zero tilt and no yaw error. The influence of the non-circulatory lift on such a pure dihedral blade is derived analytically in this section.

\subsection{Coordinate system and transformation matrix}

Following the conventions in the HAWC2 code, the main-axis of the blade is chosen to be the half-chord line. The airfoils are aligned perpendicular to this main-axis. As has been described in Sect. 2, it is necessary to perform the projection of the angular velocity and the centrifugal acceleration into the 2-D airfoil section for the analysis. As a result, it is convenient to introduce different coordinate systems and the corresponding transformation matrices between them. Three coordinate systems, which are the rotor coordinate system, the sectional coordinate system and the airfoil coordinate system are used in the present work.

We assume that the turbine has three identical blades. The rotor coordinate system corresponds to a blade that is chosen as reference. For the rotor coordinate system, the $y$-axis is following the free stream direction and is defined as the axial direction. The $z$-axis is the "radial" direction and is positive in the direction of increasing radius. The $x$-axis is defined as the tangential direction. It is normal to both $y$-axis and $z$-axis, and its direction is defined so that a right-handed system is found. The sectional coordinate system is the rotor coordinate system rotated around its $x$-axis so that the $z$-axis is tangent to the main-axis of this section and is positive in the direction of increasing curved blade length. The airfoil coordinate system 
https://doi.org/10.5194/wes-2021-163

Preprint. Discussion started: 7 January 2022

(c) Author(s) 2022. CC BY 4.0 License.

is the sectional coordinate system rotated around its $z$-axis so that the $x$-axis is in the chordwise direction, pointing from the trailing edge to the leading edge. There is only one rotor coordinate system for the whole blade, and each section has its own sectional coordinate system and airfoil coordinate system. The three coordinate systems and the relationship among them are illustrated in Fig. 3.
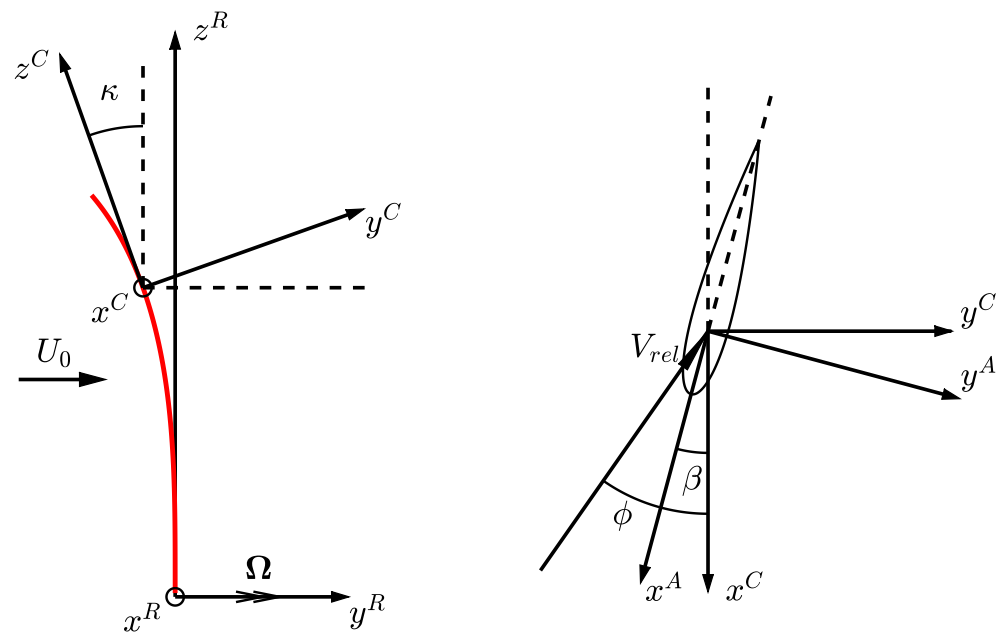

Figure 3. Illustration of the rotor coordinate system $\mathbf{R}$, the sectional coordinate system $\mathbf{C}$ and the airfoil coordinate system $\mathbf{A}$ for a blade with only dihedral and no sweep. The sectional coordinate system is the rotor coordinate system rotated around its $x$-axis with the local dihedral angle $\kappa$, as shown in the left figure. The flow angle in the sectional coordinate system is $\phi$. The airfoil coordinate system is the sectional coordinate system rotated around its $z$-axis with the twist angle $\beta$, as shown in the right figure.

For a blade section, the position vector $\boldsymbol{p}$, the angular velocity vector $\Omega$ and the centrifugal acceleration vector $\boldsymbol{a}$ in the rotor coordinate system $\mathbf{R}$ are as follows:

$\boldsymbol{p}^{\mathbf{R}}=\left(\begin{array}{c}0 \\ y \\ z\end{array}\right), \quad \boldsymbol{\Omega}^{\mathbf{R}}=\left(\begin{array}{c}0 \\ \Omega \\ 0\end{array}\right), \quad \boldsymbol{a}^{\mathbf{R}}=\left(\begin{array}{c}0 \\ 0 \\ -\Omega^{2} z\end{array}\right)$

The dihedral angle $\kappa$ is defined using the main-axis geometry in the rotor coordinate system and is defined to be positive when the blade is tilting upwind.

$\kappa=-\arctan \frac{\mathrm{d} y}{\mathrm{~d} z}$ 
The transformation matrix from the rotor coordinate system $\mathbf{R}$ to the sectional coordinate system $\mathbf{C}$ is determined by the local dihedral angle $\kappa$.

$\mathbf{T}_{\mathbf{R} \rightarrow \mathbf{C}}=\left[\begin{array}{ccc}1 & 0 & 0 \\ 0 & \cos \kappa & \sin \kappa \\ 0 & -\sin \kappa & \cos \kappa\end{array}\right]$

The twist angle $\beta$ is defined to be positive in the nose-down direction of the airfoil and the value is defined to be zero when the sectional coordinate system $\mathbf{C}$ and the airfoil coordinate system $\mathbf{A}$ are identical. For the airfoil section with the twist angle of $\beta$, the transformation matrix from the sectional coordinate system $\mathbf{C}$ to the airfoil coordinate system $\mathbf{A}$ is:

$\mathbf{T}_{\mathbf{C} \rightarrow \mathbf{A}}=\left[\begin{array}{ccc}\cos \beta & -\sin \beta & 0 \\ \sin \beta & \cos \beta & 0 \\ 0 & 0 & 1\end{array}\right]$

For a given transformation matrix, the reverse transformation matrix is equal to its transposed matrix. This is because the transformation matrices are orthonormal.

\subsection{Mid-chord acceleration}

For the pure dihedral blade, the projection of the centrifugal acceleration from the rotor coordinate system into the airfoil coordinate system is obtained using the transformation matrices in Eqs. (21) and (22).

$260 \quad \boldsymbol{a}^{\boldsymbol{A}}=\mathbf{T}_{\mathbf{C} \rightarrow \mathbf{A}} \mathbf{T}_{\mathbf{R} \rightarrow \mathbf{C}} \boldsymbol{a}^{\boldsymbol{R}}=\left(\begin{array}{c}\Omega^{2} z \sin \kappa \sin \beta \\ -\Omega^{2} z \sin \kappa \cos \beta \\ -\Omega^{2} z \cos \kappa\end{array}\right)$

According to Eq. (23) the mid-chord point of the airfoil will have an effective acceleration. The mid-chord heave acceleration is the $y$-component of $\boldsymbol{a}^{\boldsymbol{A}}$, the corresponding non-circulatory lift is obtained using Eq. (10).

$L_{\text {heav }}^{N C}=-\pi \rho b^{2} a_{y}^{A}=\pi \rho b^{2} \Omega^{2} z \sin \kappa \cos \beta$

There is also an effective streamwise acceleration that has the similar form as the negative $x$-component of $\boldsymbol{a}^{\boldsymbol{A}}$ (replacing $\beta$ with $\phi$ ) and the corresponding non-circulatory lift is derived using Eq. (10).

$L_{\text {stream }}^{N C}=-\pi \rho b^{2}(\phi-\beta) \Omega^{2} z \sin \kappa \sin \phi=L_{\text {heav }}^{N C}(\phi-\beta) \frac{\sin \phi}{\cos \beta}$

However, since the term $(\phi-\beta) \frac{\sin \phi}{\cos \beta}$ is much smaller than one, the contribution of the streamwise acceleration to the noncirculatory lift is then negligible. The total non-circulatory lift due to the projection of the centrifugal acceleration is then: 


\subsection{Airfoil effective pitching motion}

For non-planar rotors, the projection of the angular velocity into the 2-D airfoil section will also result in an effective pitching motion of the airfoil if assuming the flow seen by the airfoil is uniform. This is shown by projecting the angular velocity vector from the rotor coordinate system into the airfoil coordinate system.

$\boldsymbol{\Omega}^{\boldsymbol{A}}=\mathbf{T}_{\mathbf{C} \rightarrow \mathbf{A}} \mathbf{T}_{\mathbf{R} \rightarrow \mathbf{C}} \boldsymbol{\Omega}^{\boldsymbol{R}}=\left(\begin{array}{c}-\Omega \cos \kappa \sin \beta \\ \Omega \cos \kappa \cos \beta \\ -\Omega \sin \kappa\end{array}\right)$

The airfoil pitch rate of the effective pitching motion is the $z$-component of $\Omega^{A}$.

$\dot{\theta}=\Omega_{z}^{A}=-\Omega \sin \kappa$

The resulting non-circulatory lift due to this effective pitching motion is obtained using Eq. (10).

$L_{\text {pitchrate }}^{N C}=\pi \rho b^{2} V_{\text {rel }} \dot{\theta}=-\pi \rho b^{2} V_{\text {rel }} \Omega \sin \kappa$

\subsection{Total contribution of non-circulatory lift}

The total non-circulatory lift is then the sum of the contribution of the mid-chord acceleration in Eq. (24) and the contribution of the effective pitch rate in Eq. (29).

$L^{N C}=L_{\text {acc }}^{N C}+L_{\text {pitchrate }}^{N C}=\rho \pi b^{2} \Omega \sin \kappa\left(\Omega z \cos \beta-V_{\text {rel }}\right)$

If we assume the flow angle $\phi$ is small and the tangential induced velocity is much smaller than the velocity due to rotation, we have the following approximation:

$\Omega z \approx V_{\text {rel }} \cos \phi \approx V_{\text {rel }}$

In addition, if we assume the twist angle $\beta$ is small, the total contribution of the non-circulatory lift for an pure dihedral blade in steady-state operating condition is then approximately zero.

$L^{N C} \approx \rho \pi b^{2} \Omega \sin \kappa\left(V_{\text {rel }}-V_{\text {rel }}\right)=0$

Since the non-circulatory lift is perpendicular to the airfoil, there should be an effective non-circulatory drag $D^{N C}$ that corresponds to the flow direction due to the projection of the non-circulatory lift as shown in Sect. 2.2.2. However, since $L^{N C}$ is approximately zero, this effective drag $D^{N C}$ is also approximately zero. In summary, for a pure dihedral blade operating at a steady-state condition, the total non-circulatory lift and the corresponding effective non-circulatory drag are negligible. This conclusion will be tested numerically in Sect. 7.1. Since a VAWT is similar to a HAWT with a $90^{\circ}$ cone when there is no inflow, it is not surprising that the same conclusion has been shown for a VAWT operating at steady-state conditions in Pirrung and Gaunaa (2018) as well as in Sect. 2.2.4. 
It is important to note that the equations derived previously in this section are only applicable to a pure dihedral blade without sweep under steady-state operating conditions. For unsteady cases, there could be net non-circulatory forces. As a result, for the unsteady conditions, it is important to include all the non-circulatory terms described in Sect. 2, for both the lift and drag forces.

\section{One-point lifting-line correction}

According to the conclusions from the unsteady 2-D thin airfoil theory described in Sect. 2, the generalized lifting-line method should be implemented using the flow information at two chordwise locations: the magnitude of the quasi-steady lift should be determined using the angle of attack at the three-quarter-chord point, and the direction of the quasi-steady lift should be determined using the flow angle at the quarter-chord point. This means the projection of the quasi-steady lift and drag coefficients into the sectional coordinate system, as usually done in many BEM literature, should have the following form ${ }^{4}$ :

$C_{x}^{Q S}=C_{L}^{Q S}\left(\alpha_{3 / 4}\right) \sin \phi_{1 / 4}-C_{D}^{Q S}\left(\alpha_{3 / 4}\right) \cos \phi_{1 / 4}$

$C_{y}^{Q S}=C_{L}^{Q S}\left(\alpha_{3 / 4}\right) \cos \phi_{1 / 4}+C_{D}^{Q S}\left(\alpha_{3 / 4}\right) \sin \phi_{1 / 4}$

where

$\phi_{1 / 4}=\alpha_{1 / 4}+\beta$

The generalized lifting-line methods are usually implemented as the one-point approach that only utilizes the flow information at one chordwise location per section for simplicity. This simplification will not change the performance prediction of planar rotors, because the flow angle is constant along the chord for planar rotors under steady-state conditions. This can be shown using Eq. (28) with the condition of $\kappa=0$, there is no effective pitching motion of the airfoil. However, there exists an effective pitching motion for the airfoil section of a non-planar rotor even in steady-state conditions, as shown in Sect. 3.3. So the simplified one-point approach will result in an error to the performance prediction of non-planar rotors.

However, with the known effective pitch rate $\dot{\theta}$, the flow angle at the quarter-chord point and the three-quarter-chord point can be inferred from each other, or from the known flow angle at an arbitrary chordwise location. For example, the difference between the flow angle at the quarter-chord point and at the three-quarter-chord point can be approximated using Eq. (5).

$\phi_{3 / 4}-\phi_{1 / 4}=\alpha_{3 / 4}-\alpha_{1 / 4}=\arcsin \frac{w_{3 / 4}}{V_{\mathrm{rel}, 3 / 4}}-\arcsin \frac{w_{1 / 4}}{V_{\mathrm{rel}, 1 / 4}} \approx \frac{\dot{\theta} c}{2 V_{\text {rel }}}$

The difference between the magnitude of the relative velocity at the three-quarter-chord point $\left(V_{\text {rel, } 3 / 4}\right)$ and at the quarterchord point $\left(V_{\text {rel,1/4 }}\right)$ is a secondary effect, which will be shown numerically in Sect. 7.2. In the following sections, the magnitude of the relative velocity $V_{\text {rel }}$ along the chord is assumed to be constant, unless otherwise stated. For the special case that a pure dihedral blade is operating at a steady-state condition, Eq. (36) can be simplified using Eq. (28).

$\alpha_{3 / 4}-\alpha_{1 / 4} \approx-\frac{\Omega c \sin \kappa}{2 V_{\text {rel }}}$

\footnotetext{
${ }^{4}$ It is argued by some researchers that the drag force should be excluded during the convergence calculation, but should be included when calculating the aerodynamic loads after the convergence, which can be considered as the post-processing of the converged results.
} 


\subsection{One-point approach using the quarter-chord point}

One of the common one-point implementations of the generalized lifting-line method is placing the calculation point at the quarter-chord point for each section. The flow information at the quarter-chord point is used to determine both the magnitude and direction of the lift force. An example of this is the most common implementation of the lifting-line (LL) method (Phillips and Snyder, 2000). For a dihedral blade, the directions of the lift and drag forces from this simplified one-point approach are correct, but the magnitude of the forces will be wrong. The correction to this implementation is using the approximated angle of attack at the three-quarter-chord point in Eq. (38) instead of the angle of attack at the quarter-chord point, to obtain the quasi-steady aerodynamic coefficients from the 2-D airfoil data.

$\tilde{\alpha}_{3 / 4} \approx \alpha_{1 / 4}+\frac{\dot{\theta} c}{2 V_{\text {rel }}}$

\subsection{One-point approach using the three-quarter-chord point}

Another commonly used one-point approach of the generalized lifting-line methods is placing the calculation point at the three-quarter-chord point, such as the BEM method implemented in the HAWC2 code. This implementation uses the flow information at the three-quarter-chord point for both the magnitude and direction of the lift force. Then, the magnitude of the circulatory lift and drag coefficients are correctly calculated, but the direction of the lift force is erroneous, which will result in an additional effective drag force. Then, the calculated tangential load will have an offset if the blade has dihedral, which will result in an error in the aerodynamic power prediction.

One possible method of applying the correction is using the angle of attack at the three-quarter-chord point and the pitch rate $\dot{\theta}$ to approximate the angle of attack at the quarter-chord point as in Eq. (39). Then, the approximated value of $\tilde{\alpha}_{1 / 4}$ should be used in Eq. (35) to determine the directions of the lift and drag.

$\tilde{\alpha}_{1 / 4} \approx \alpha_{3 / 4}-\frac{\dot{\theta} c}{2 V_{\text {rel }}}$

Alternatively, it is possible to include an additional pitch rate drag force in the flow direction at the three-quarter-chord point by modifying the quasi-steady drag coefficient in Eqs. (33) and (34). This formulation of the one-point lifting-line correction is implemented in the Beddoes-Leishman type dynamic stall model in HAWC2 code version 12.5 and later (Pirrung and Gaunaa, 2018).

$$
\begin{aligned}
\tilde{C}_{D}^{Q S} & =C_{D}^{Q S}\left(\alpha_{3 / 4}\right)+C_{L}^{Q S}\left(\alpha_{3 / 4}\right)\left(\alpha_{3 / 4}-\tilde{\alpha}_{1 / 4}\right) \\
& =C_{D}^{Q S}\left(\alpha_{3 / 4}\right)+\frac{\dot{\theta} c}{2 V_{\mathrm{rel}}} C_{L}^{Q S}\left(\alpha_{3 / 4}\right)
\end{aligned}
$$

Both implementations should give almost identical results when the difference between $\alpha_{3 / 4}$ and $\tilde{\alpha}_{1 / 4}$ is small.

\subsection{Generalized one-point correction}

Apart from the two most common choices of the calculation point described previously, other definitions of the calculation point are possible. The general correction for the one-point approach of the generalized lifting-line methods that use an arbitrary 
chordwise location as the calculation point is given. When placing the calculation point at the $x$-chord point, which means the distance from the leading edge to the calculation point is $x c$, there should be corrections to both the magnitude and direction of the lift force. This can be done by approximating the angle of attack at both the quarter-chord point and the three-quarter-chord point from the angle of attack at the $x$-chord point together with the pitch rate $\dot{\theta}$ according to Eq. (5).

$\tilde{\alpha}_{3 / 4}=\alpha_{x}+\left(\frac{3}{4}-x\right) \frac{\dot{\theta} c}{V_{\text {rel }}}$

$\tilde{\alpha}_{1 / 4}=\alpha_{x}+\left(\frac{1}{4}-x\right) \frac{\dot{\theta} c}{V_{\text {rel }}}$

The approximated angle of attack $\tilde{\alpha}_{3 / 4}$ should be used in Eqs. (33) and (34) to obtain the quasi-steady lift and drag coefficient from the airfoil data. The approximated angle $\tilde{\alpha}_{1 / 4}$ should be used in Eq. (35) to determine the direction of the lift and drag forces.

\section{Blade geometries for comparison}

The blades used for the numerical tests in the present work are based on the IEA-10.0-198 $10 \mathrm{MW}$ reference wind turbine (RWT) (Bortolotti et al., 2019). Two different blades are used, which are the baseline straight blade and an upwind dihedral blade. The baseline straight blade is modified by removing the prebend and sweep of the original blade of the RWT so that the main-axis of the half-chord line is aligned into a straight line. The upwind dihedral blade is modified from the baseline straight blade so that the half-chord line has out-of-plane shapes. The geometry of the upwind dihedral blade is identical to the W-1 blade in a previous study ( $\mathrm{Li}$ et al., 2021). The blade has the same radius as the straight blade but is bending upwind from 50\% of radius until the blade tip. The dihedral magnitude is $10 \%$ at the blade tip and the tip dihedral angle is $20^{\circ}$.

In the present work, the test cases are mostly using the baseline straight blade with zero coning, $15^{\circ}$ upwind coning and $15^{\circ}$ downwind coning. In addition selected results are presented for an extreme case, represented by the upwind dihedral blade W- 1 with added $15^{\circ}$ of upwind coning. For the different cases, the main-axes of the blades used for the comparison are illustrated in Fig. 4. For the rotor with straight blades and without cone, the radius is $99 \mathrm{~m}$, of which the hub radius is $2.8 \mathrm{~m}$. 

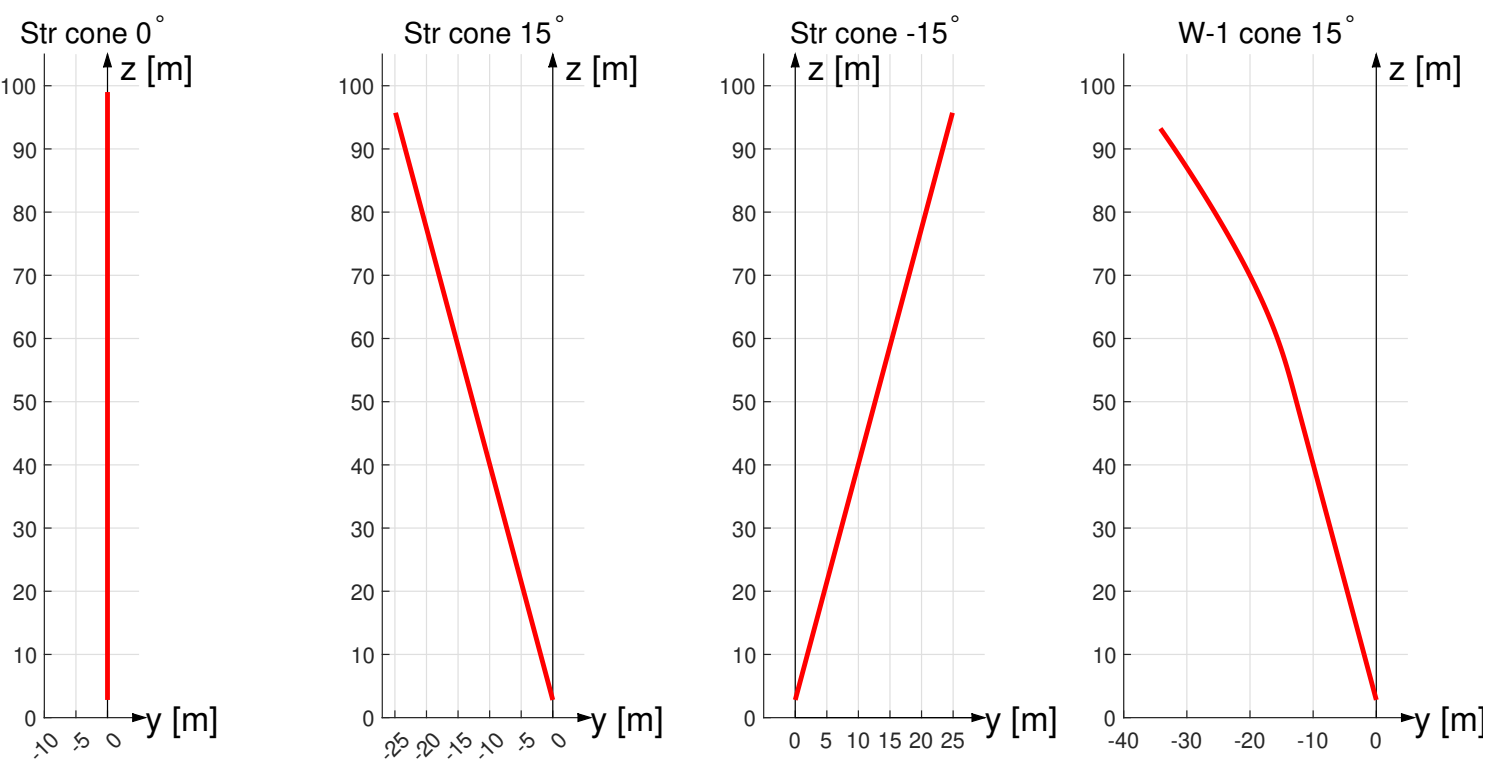

Figure 4. Side-view of the main-axes of the blades used for different cases of the comparison. The blades from left to right are the baseline straight blade with zero cone, with $15^{\circ}$ upwind coning, $15^{\circ}$ downwind coning and the upwind dihedral blade $\mathrm{W}-1$ with added $15^{\circ}$ of upwind coning.

\section{The aerodynamic models for comparison}

In this section, the different aerodynamic models with different numerical fidelities used for comparison are described. The highest-fidelity model used for the comparison is based on blade geometry resolving RANS simulations. The generalized lifting-line methods with different fidelities are compared, which are the actuator line (AL) method, the lifting-line (LL) method, the BEVC method and the BEM method. The airfoil data used in all generalized lifting-line methods in the present work are the same, and they were generated with 2-D fully turbulent RANS computations (Bortolotti et al., 2019).

\subsection{Fully-resolved Navier-Stokes simulations}

For the numerical simulations that will be used as a reference in the present study, the three-dimensional computational fluid dynamics (CFD) solver EllipSys3D (Michelsen, 1992, 1994; Sørensen, 1995) was used. EllipSys3D solves the incompressible Navier-Stokes equations based on a finite volume discretization, using structured meshes and a multi-block strategy. A RANS turbulence modeling approach was employed in the present study, relying on the k- $\omega$ SST model (Menter, 1994). For these fully-resolved simulations, body-fitted grids were built around the surface of each of the studied blade geometries, and a wall boundary condition was imposed. Each surface grid was generated using the open-access Parametric Geometry Library (PGL) (Zahle, 2019). The resolution of the surface grids was $256 \times 128$ (corresponding to the chordwise direction and the spanwise direction, respectively). Starting from those surface meshes, hyperbolic volume grids were generated with the in-house tool 
Hypgrid (Sørensen, 1998). A total of 256 cells were generated when marching to the outer limit of the CFD domain, which is located at approximately 11 radii from the surface grid. A boundary layer clustering with an initial cell height of $1 \times 10^{-6} \mathrm{~m}$ was used, in order to target wall-resolved simulations. Each of the resulting volume meshes had a total of 14.2 million cells. The flow was assumed to be fully turbulent, and an inlet/outlet approach was followed for the boundary conditions of the outer domain. Throughout this document, the body-fitted RANS simulations described here are referred to as fully-resolved CFD, or simply CFD. In order to illustrate the mesh topology, Fig. 5 shows a superposition of the grid for the straight blade variants with cone angles of $0^{\circ}$ and $15^{\circ}$ (upwind).

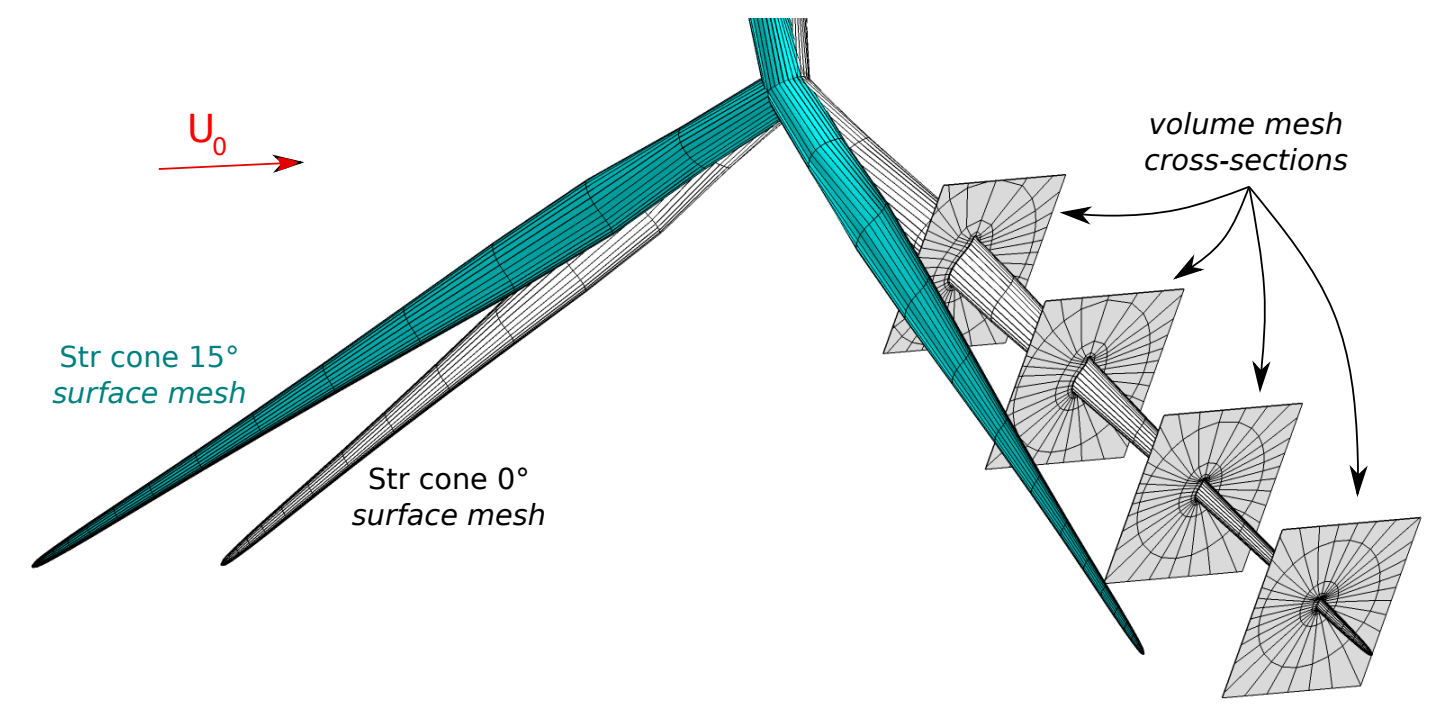

Figure 5. Detail of the surface mesh for the straight variants used in the fully-resolved CFD simulations, showing two of the blades. Two different configurations are included, accounting for a cone angle of $0^{\circ}$ and $15^{\circ}$. For the zero cone angle case, the topology of the volume mesh is shown through arbitrary cross-sectional cuts along the span. The free-stream velocity vector is $U_{0}$. For clarity, only every eighth grid line is shown.

\subsection{Lifting-line method}

The lifting-line module in the aerodynamic solver MIRAS (Ramos-García et al., 2016) is used for comparison. The numerical lifting-line (LL) solver is implemented as a free-wake vortex method in a time-marching fashion. The curved bound vortex influence is modeled by including the difference between the 3-D bound vortex induction and the 2-D bound vortex induction evaluated at the three-quarter-chord point ( $\mathrm{Li}$ et al., 2020). It should be emphasized that the induced velocity vector due to the curved bound vortex is assumed to be constant along the chord. For the simulations, each time step corresponds to $1.5^{\circ}$ of azimuthal rotation. Each simulation is calculated for 20 thousand time steps which correspond to 83.3 revolutions. The vortex core size is chosen to be $0.1 \%$ of the local chord length. Each blade of the rotor is discretized radially into 50 sections with cosine spacing. Both one-point and two-point approaches of the LL method are implemented. 


\subsubsection{Two-point approach}

The two-point approach in the LL method refers to the explicit calculation of the flow information at both the quarter- and the three-quarter-chord point and is labeled as LL-2P. This approach aligns with the conclusions from the unsteady 2-D thin airfoil theory, as previously described in Sect. 2. The angle of attack at the three-quarter-chord point is used to determine the magnitude of the lift and drag coefficients, whereas the angle of attack at the quarter-chord point is used to determine the direction of lift and drag.

\subsubsection{One-point approach}

The one-point approaches only use the flow information at a single chordwise location for each section. Two variants of the one-point approach, which are representative of the most common implementations, will be used for the comparison. The first one is using only the quarter-chord point as the calculation point (labeled as LL-QC), the second one is using only the three-quarter-chord point (labeled as LL-3QC). The corrections previously described in Sect. 4.1 and 4.2 are implemented for each of the one-point LL methods, respectively. Results corrected accordingly are subsequently labeled as LL-QC-corr and LL-3QC-corr. For LL-3QC-corr, the correction is implemented by including the additional pitch rate drag force as presented in Eq. (40).

\subsection{BEM method}

The blade element momentum (BEM) method implemented in the HAWC2 code is the one-point approach that only uses the three-quarter-chord point as the calculation point (Madsen et al., 2020a). The one-point lifting-line correction that is implemented as an additional effective pitch rate drag force described in Sect. 4.2 is already included in the Beddoes-Leishman type dynamic stall model (Hansen et al., 2004; Pirrung and Gaunaa, 2018) in HAWC2. For the steady-state case, the non-circulatory force has negligible influence, which has been shown analytically in Sect. 3 and will be shown numerically in Sect. 7.1. As a result, the HAWC2 BEM with the dynamic stall model enabled is similar to the one-point approach of the LL method with the calculation point placed at the three-quarter-chord line and with the correction (LL-3QC-corr). The HAWC2 BEM that directly uses the quasi-steady aerodynamics is similar to the one-point approach of the LL method with the calculation point placed at the three-quarter-chord line but without the correction (LL-3QC).

\subsection{BEVC method}

The blade element vortex cylinder (BEVC) method is the combination of the blade element theory and the vortex cylinder model. It has been shown in a previous work ( $\mathrm{Li}$ et al., 2021) that the distributed loads of dihedral blades predicted by the BEVC model agree significantly better with higher-fidelity LL and fully-resolved CFD, than BEM results. In that previous work, it was described that the unsteady airfoil aerodynamic effects are included in the numerical tests, which is actually done by enabling the Beddoes-Leishman type dynamic stall model when implemented in the HAWC2 code. This means the steadystate results in that previous work already included the one-point lifting-line correction described in Sect. 4. In the present 
https://doi.org/10.5194/wes-2021-163

Preprint. Discussion started: 7 January 2022

(c) Author(s) 2022. CC BY 4.0 License.

(c) (i)

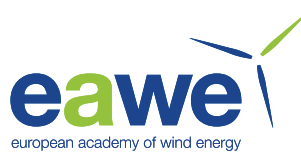

work, the results with and without the correction will be compared to show the importance of the effect. Similar to the HAWC2 BEM module, the BEVC method implemented in HAWC2 also uses the three-quarter-chord point as the calculation point.

\subsection{Actuator line simulations}

Consistent with the fully-resolved CFD computations, the actuator line (AL) simulations also used the EllipSys3D flow solver ${ }^{5}$ with the same numerical setup as the fully-resolved simulations described in Sect. 6.1. However, instead of employing a body-fitted rotor mesh, the AL (Sørensen and Shen, 2002) distributes the sectional blade forces-computed from 2-D airfoil polars-over a Cartesian box mesh by a three-dimensional Gaussian kernel. This base formulation is enhanced by including the computationally efficient smearing correction described by Meyer Forsting et al. (2019a, b, 2020). Such a correction renders the AL equivalent to a lifting-line, as proven by Meyer Forsting et al. (2019a); Martínez-Tossas and Meneveau (2019). Without the correction, the AL acts as a LL with a viscous core. In the present work, the smearing correction is partially assuming the rotor to be planar due to the underlying assumptions in the near-wake model (Pirrung et al., 2016).

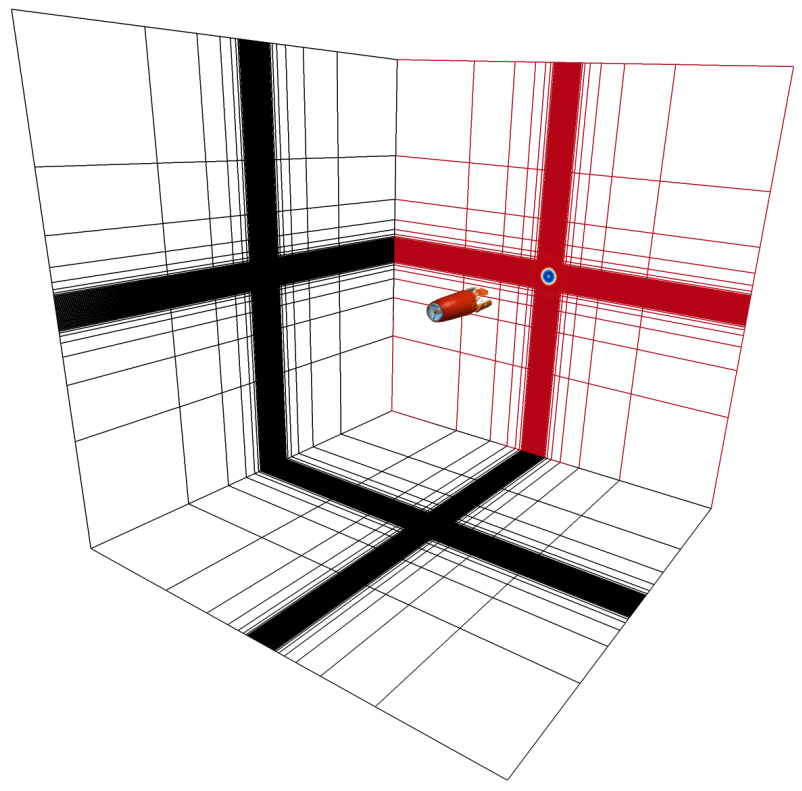

Figure 6. Actuator line numerical box domain with a structured mesh and uniform spacing around the rotor at its center. Only every eighth grid point is shown.

The numerical domain discretization follows a verified approach (Meyer Forsting et al., 2017; Troldborg et al., 2009) and consists of a box with $50 R(R=99 \mathrm{~m})$ edge lengths that contains a rectangular, uniformly spaced refined mesh with $3.2 R$ side lengths in the cross-flow and $3.9 R$ in the streamwise direction. The rotor is placed in the domain center, with the refined

\footnotetext{
${ }^{5}$ Even if the described simulations are based on a CFD solver, they are simply referred to as AL in this document-keeping the CFD acronym for the fully-resolved computations.
} 
mesh starting 1.6R upstream, as illustrated in Fig. 6. The latter thus surrounds the AL and has a grid spacing of $\Delta x=R / 80$ to resolve the flow features of interest. The full volume mesh has 75 million cells. The domain boundaries off the main flow direction are of the Symmetry type, whereas the inflow and outflow faces obey Dirichlet and Neumann conditions, respectively. To ensure the blade tip to remain well inside a single grid cell during each time step, $\Delta t=0.0117 \mathrm{~s}$. The smearing or kernel length scale is twice the grid size as recommended by Troldborg et al. (2009) to guarantee numerical stability and the blade is discretized into 100 aerodynamic sections between root and tip. The one-rotation averaged sectional blade forces are converged down to a residual of $1 \times 10^{-5}$.

In the AL method, the velocity is only calculated on the actuator line itself. Considering the equivalence between the AL and the LL method for straight blades without cone, the AL method before the one-point correction is similar to the LL method that only uses the quarter-chord locations in the load computations (LL-QC), as described in Sect. 6.2.2. Similarly, the AL method following the one-point correction should be equivalent to LL-QC-corr.

\section{Results}

In this section, various numerical tests are performed to investigate the different assumptions outlined in the previous sections and also to evaluate the performance of the one-point lifting-line correction for different aerodynamic models. Firstly, the impact of non-circulatory forces at steady-state is tested using the LL method in Sect. 7.1. Then, the impact of the variation of the relative velocity magnitude along the chord is investigated numerically in Sect. 7.2. Afterwards, the one-point lifting-line correction is tested for different implementations of the LL method in Sect. 7.3, for the BEM method in Sect. 7.4, for the BEVC method in Sect. 7.5 and for the AL method in Sect. 7.6.

For all test cases, the pitch angle is zero and the rotor is operating under uniform inflow of $8 \mathrm{~m} \mathrm{~s}^{-1}$ that is perpendicular to the rotor plane. The rotational speed of the rotor is constant at $0.855 \mathrm{rad} \mathrm{s}^{-1}$. For the cases with zero cone angle, the radius is $99 \mathrm{~m}$ and the tip speed ratio is 10.58 . At this operational condition, the thrust coefficient of the unconed rotor with baseline straight blades is 0.90 and the rotor power coefficient is 0.46 , as predicted using the BEM method.

The initial assessment of the performance of the different numerical methods relies on the study of the sectional aerodynamic load distributions. In order to ensure the quality of the comparison among different blade geometries involved in the present study, it is important to define the loads in a consistent manner. The loads are defined as force per unit radius, which is the same definition used in the previous work ( $\mathrm{Li}$ et al., 2021). The sectional aerodynamic loads that are calculated from the 2-D airfoil data and projected into the rotor coordinate system should correspond to force per unit curved blade length. The loads with the definition of force per unit radius are obtained by multiplying the curved blade length correction factor $\mathrm{d} s / \mathrm{d} r$ (Madsen et al., 2020a). For the coned straight blade case, the correction factor is equal to the secant of the cone angle. For the dihedral blade with added coning, the correction factor is equal to the secant of the sum of the cone angle and the local dihedral angle (Li et al., 2021). 


\subsection{The non-circular force at steady-state}

It has been shown analytically in Sect. 3.4 that the total non-circulatory lift of a pure dihedral blade operating at steady-state conditions under uniform inflow perpendicular to the rotor plane is approximately zero. In addition, it has been shown in Sect. 2.2.3 that there is a non-circulatory drag force due to the pitch rate caused by the effective pitch rate of the airfoil. It was analyzed that the contribution is also negligible for low to moderate reduced frequency cases. In this section, these two conclusions are tested numerically. This is done by comparing the aerodynamic loads of a pure dihedral blade calculated from the two-point approach of the LL method without non-circulatory forces (LL-2P), with non-circulatory lift (LL-2P-NC1) and with both non-circulatory lift and non-circulatory pitch rate drag (LL-2P-NC2). As has been described previously, the noncirculatory lift is perpendicular to the airfoil. So, there will be an effective non-circulatory drag in the flow direction due to the projection of the non-circulatory lift, which is included in both LL-2P-NC1 and LL-2P-NC2. The extreme case of the upwind dihedral blade $\mathrm{W}-1$ with additional $15^{\circ}$ of upwind coning is used here, to amplify the influence of blade dihedral on the non-circulatory forces. The axial and tangential loads predicted by the two-point LL approach, both with and without the non-circulatory forces are compared in Fig. 7. In addition, the fully-resolved CFD results are included as reference.
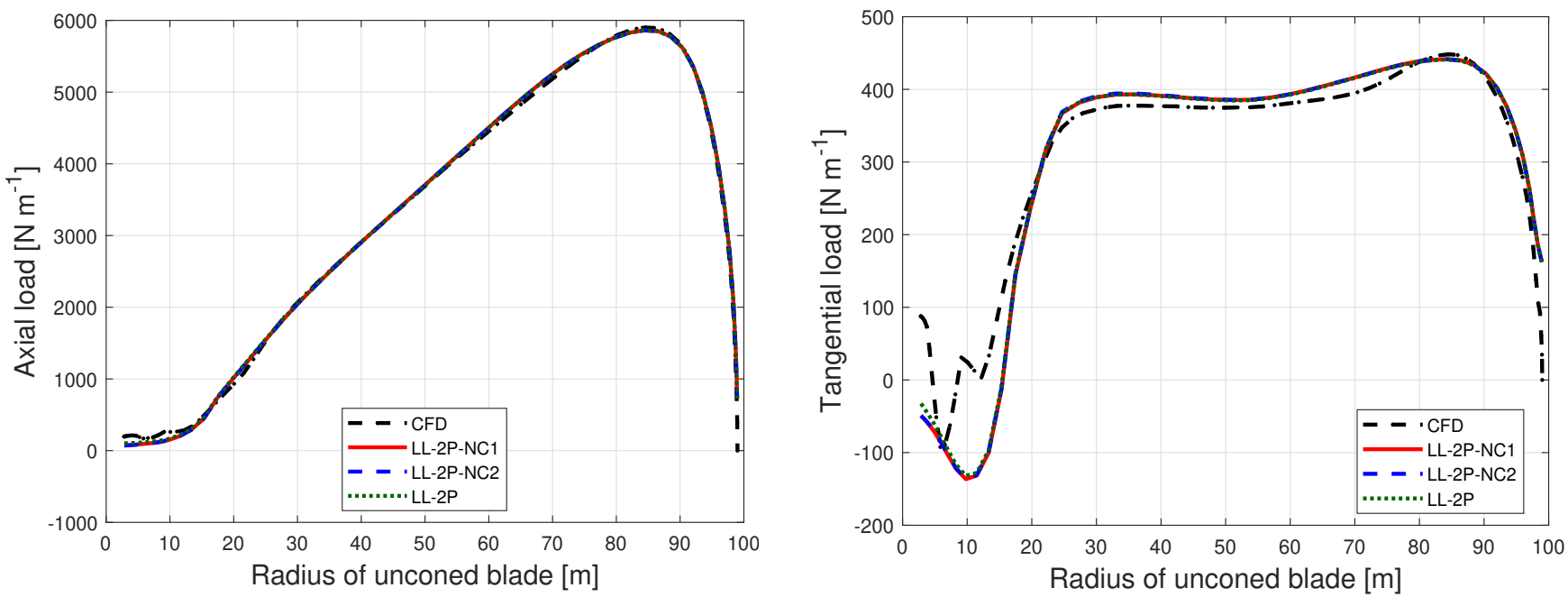

Figure 7. Comparison of axial load (left) and tangential load (right) of the upwind dihedral blade W-1 with additional $15^{\circ}$ of upwind coning calculated from the fully-resolved CFD, the two-point approach of the LL method without non-circulatory force (LL-2P), with noncirculatory lift (LL-2P-NC1) and with both non-circulatory lift and the non-circulatory pitch rate drag (LL-2P-NC2).

It can be seen from the figure that the loads from the LL method with or without the non-circulatory force are almost identical and are in good agreement with the results from the fully-resolved CFD solver. To clearly show the magnitude of the non-circulatory forces, the difference of the loads calculated from LL-2P-NC1 and LL-2P-NC2 with respect to the results from LL-2P are calculated. The difference generally increases when moving from the blade tip to the blade root but is negligible. For the spanwise position between radius of $40 \mathrm{~m}$ (of the unconed blade) to the blade tip, the difference compared to the LL-2P method is within $1.5 \mathrm{~N} \mathrm{~m}^{-1}$ for axial load and is within $5 \mathrm{~N} \mathrm{~m}^{-1}$ for the tangential load. For the radius of $20 \mathrm{~m}$ (of the unconed 
blade), the difference is $2 \mathrm{~N} \mathrm{~m}^{-1}$ for axial load and is $12 \mathrm{~N} \mathrm{~m}^{-1}$ for the tangential load. It should be emphasized that the total non-circulatory force may not be negligible for unsteady cases.

\subsection{The variation of relative velocity magnitude}

For the generalized lifting-line methods, there are two procedures that involve the magnitude of the relative velocity for the calculation. The first one is the calculation of the quasi-steady bound circulation strength, which is related to the convergence calculation.

$\Gamma=\frac{1}{2} V_{\mathrm{rel}} c C_{L}^{Q S}\left(\alpha_{3 / 4}\right)$

The second one is during the calculation of the lift and drag forces, which is to compute the aerodynamic loads on the blades.

510 This procedure is performed after the convergence calculation and can be considered as the post-processing of the converged results.

$$
\begin{aligned}
L^{Q S} & =\frac{1}{2} \rho V_{\mathrm{rel}}^{2} c C_{L}^{Q S}\left(\alpha_{3 / 4}\right) \\
D^{Q S} & =\frac{1}{2} \rho V_{\mathrm{rel}}^{2} c C_{D}^{Q S}\left(\alpha_{3 / 4}\right)
\end{aligned}
$$

There is no clear indication from unsteady thin airfoil theory at which chordwise location to extract the relative velocities

515 for any of these two procedures. For the one-point approach, it is natural to use the relative velocity at the calculation point for both procedures. For the two-point approach, it is possible to choose either the relative velocity at the quarter-chord point or at the three-quarter-chord point for both procedures. In total, there are four possible combinations and are summarized in Table 1.

Table 1. The four different choices of the chordwise location for the magnitude of the relative velocity to use for the calculation of the bound circulation strength and the magnitude of the lift and drag force in the two-point approach of the lifting-line method.

\begin{tabular}{lll}
\hline \hline Name & Bound circulation strength & Magnitude of lift and drag \\
\hline Case 1 & $1 / 4$ chord & $1 / 4$ chord \\
Case 2 & $1 / 4$ chord & $3 / 4$ chord \\
Case 3 & $3 / 4$ chord & $1 / 4$ chord \\
Case 4 & $3 / 4$ chord & $3 / 4$ chord \\
\hline \hline
\end{tabular}

From an intuitive point of view, Case 3 appears as the most correct one because the angle of attack at the three-quarterchord point is used to determine the lift coefficient and the flow at the quarter-chord point is used to determine the lift and drag direction. The difference between these four combinations is tested numerically by comparing the loads calculated using different implementations of LL-2P. For this numerical test, the extreme case with the upwind dihedral blade W- 1 with $15^{\circ}$ 

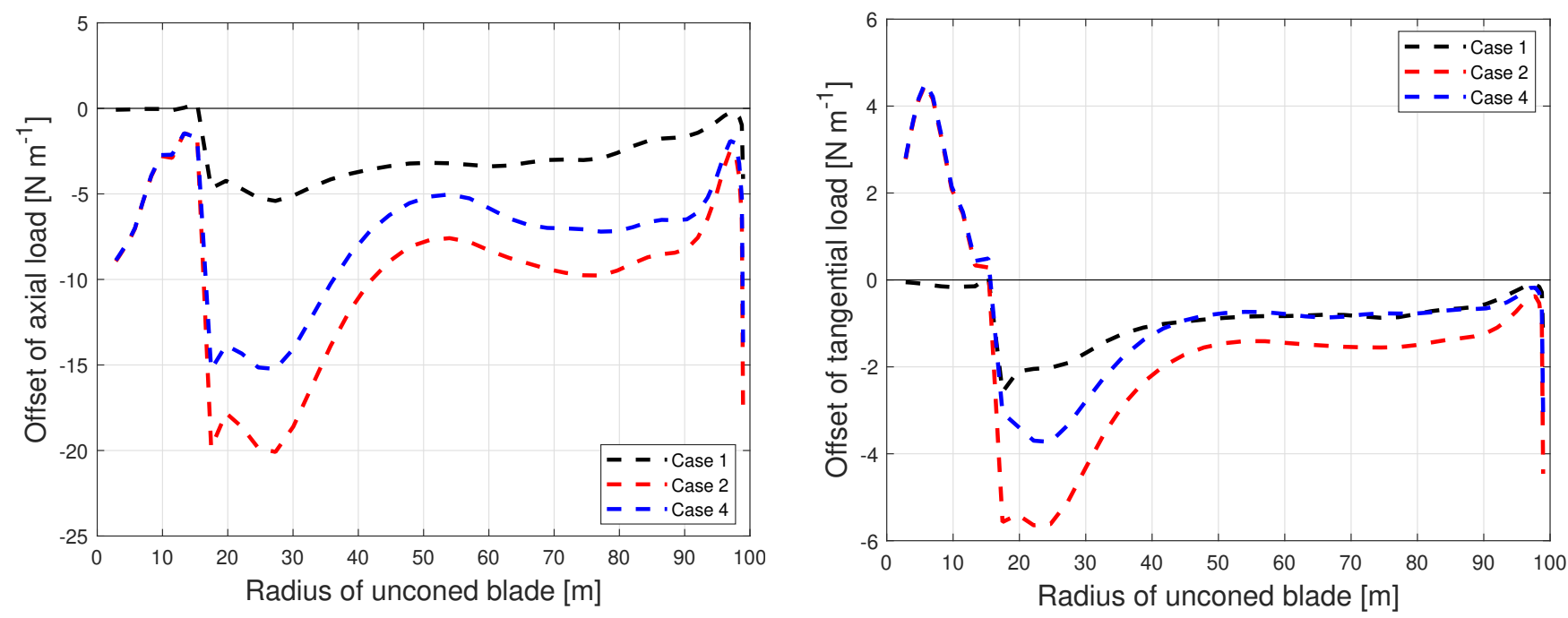

Figure 8. Comparison of the difference of axial load (left) and tangential load (right) of the upwind dihedral blade $\mathrm{W}-1$ with $15^{\circ}$ of additional upwind coning calculated using the three different methods compared to the intuitive correct method (Case 3) from the two-point LL methods.

of additional upwind coning is used. To clearly show the difference between different implementations, the difference of the loads of the other three methods compared to the intuitively most correct method (Case 3) are plotted in Fig. 8.

It can be seen that the difference of the loads calculated using different methods is extremely small compared to the full loads as shown in Fig. 7 and are thus negligible. For example, at the spanwise location that corresponds to the $70 \mathrm{~m}$ radius of the unconed blade, the maximum difference is less than $0.2 \%$ for the axial load and is less than $0.4 \%$ for the tangential load. It is then confirmed numerically that the variation of the relative velocity magnitude along the chord for the calculation of bound circulation and the magnitude of lift and drag forces is a secondary effect. Then, for the two-point approach, the choice of the relative velocity for the two procedures can be arbitrary. The conclusion can also justify that the one-point approach directly uses the relative velocity at the calculation point for both procedures.

\subsection{One-point lifting-line corrections}

The correction to the generalized one-point lifting-line method described in Sect. 4 is tested numerically using the LL method in this section. The straight blade with $15^{\circ}$ of cone upwind or downwind is used here. In addition, the case of the straight blade without cone is also included as reference to show the influence of blade coning.

The two-point approach of the LL method (LL-2P) ${ }^{6}$ is used as the reference method since it coincides with the conclusions from the unsteady 2-D thin airfoil theory. In addition, the fully-resolved CFD results are included for reference. Two different simplified one-point approaches of the LL method (LL-QC and LL-3QC), with the calculation point either at the quarter- or three-quarter-chord line are compared. As has been described in Sect. 4.1 and 4.2, the two methods calculate the wrong magnitude of the lift force and apply the lift force in the wrong direction, respectively. The previous two methods with the corrections

\footnotetext{
${ }^{6}$ The LL-2P method in the following sections corresponds to Case 3 in Table 1.
} 
are LL-QC-corr and LL-3QC-corr. The numerical tests are performed by comparing the aerodynamic loads calculated from these different implementations of the LL methods as well as the fully-resolved CFD.

\subsubsection{Straight blade without cone}

Firstly, the axial and tangential loads of the straight blade without cone calculated from different implementations of the LL method are calculated and are plotted together with the fully-resolved CFD results in Fig. 9.
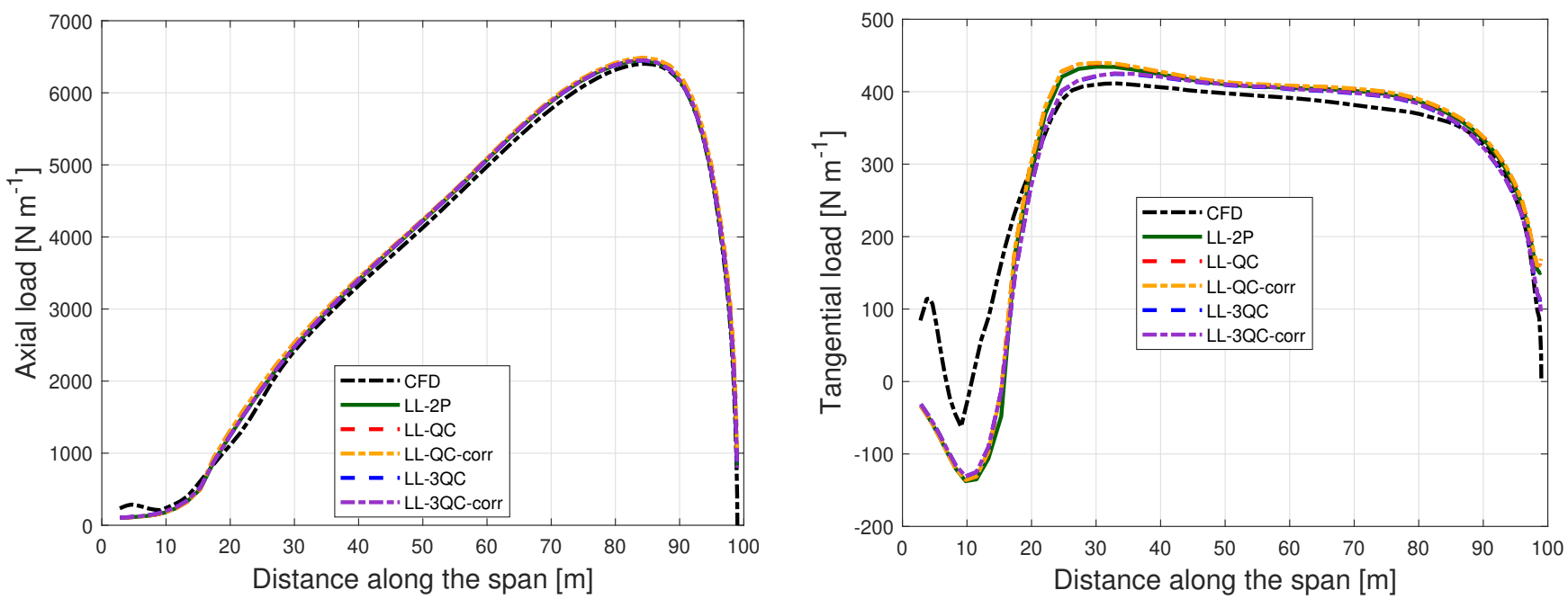

Figure 9. Comparison of axial load (left) and tangential load (right) of the straight blade without cone calculated from different LL methods and the fully-resolved CFD.

It can be seen from the figure that the loads from all LL methods give very similar results for both axial and tangential loads. The results in the figures are almost on top of each other. The loads predicted by the LL methods are also in good agreement with the CFD results.

\subsubsection{Upwind coned case}

The axial and tangential loads of the straight blade with $15^{\circ}$ of upwind coning are calculated from different implementations of the LL method and are plotted together with the fully-resolved CFD results in Fig. 10.

It can be seen from the figure that for the axial load, all LL methods except LL-QC give very similar results, and are in good agreement with the fully-resolved CFD. The axial load is overestimated by LL-QC. For the tangential load, the LL3QC method predicts a somewhat lower value compared to the other LL methods, while the other methods show only small differences and are in good agreement with the fully-resolved CFD. To better illustrate the effect of blade coning predicted by different LL methods, the difference of the loads of the coned straight blade with respect to the baseline straight blade without cone are plotted in Fig. 11. 

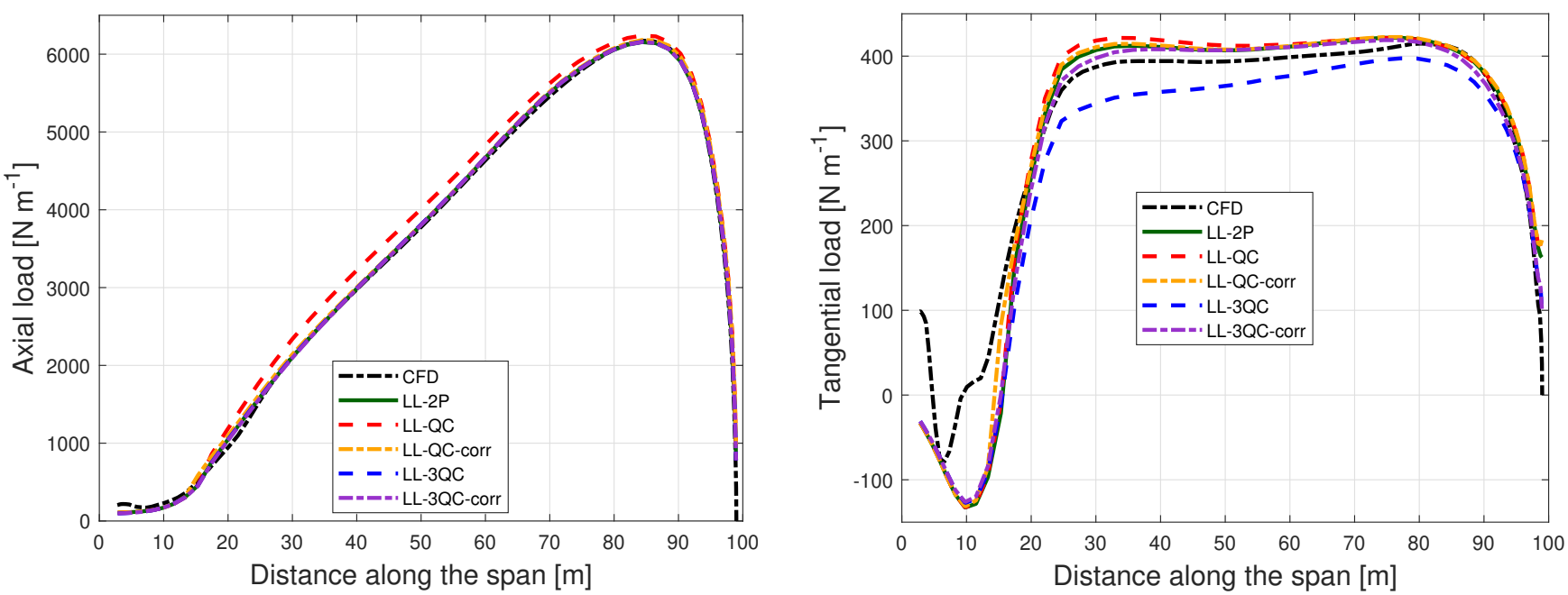

Figure 10. Comparison of axial load (left) and tangential load (right) of the straight blade with $15^{\circ}$ of upwind coning calculated from different LL methods and the fully-resolved CFD.
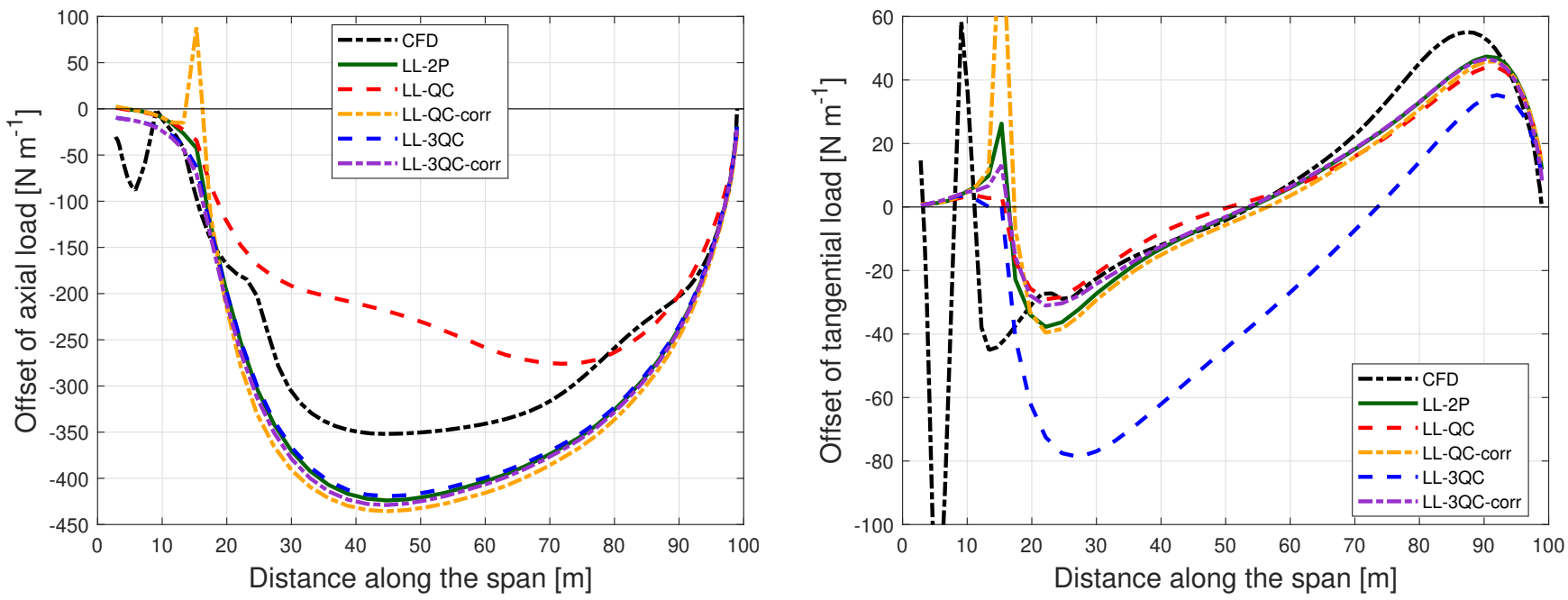

Figure 11. Comparison of the difference of the axial load (left) and tangential load (right) of the straight blade with $15^{\circ}$ of upwind coning compared to the straight blade with zero cone, calculated from different implementations of the LL method and the fully-resolved CFD. 
For the LL-QC method, the decrement of the axial load is significantly underestimated compared to the predictions by the LL-2P. This is expected, since the magnitude of the lift is not correctly calculated using the LL-QC method. After applying the correction, the axial load from LL-QC-corr agrees significantly better with LL-2P. For the tangential load, the result from LL-QC is in reasonably good agreement with the other methods, as despite the magnitude of the lift force having an offset, the lift force is applied in the correct direction.

For the LL-3QC method, the calculated axial load is in good agreement with the result from LL-2P because the magnitude of the lift force is correctly calculated. The tangential load calculated from LL-3QC is underestimated compared to LL-2P. This is because the lift force is not applied to the correct direction in LL-3QC. After applying the correction, the tangential load predicted by LL-3QC-corr is in good agreement with LL-2P.

\subsubsection{Downwind coned case}

For the straight blade with $15^{\circ}$ of downwind coning, the axial and tangential loads calculated from different LL methods and the fully-resolved CFD are plotted in Fig. 12.
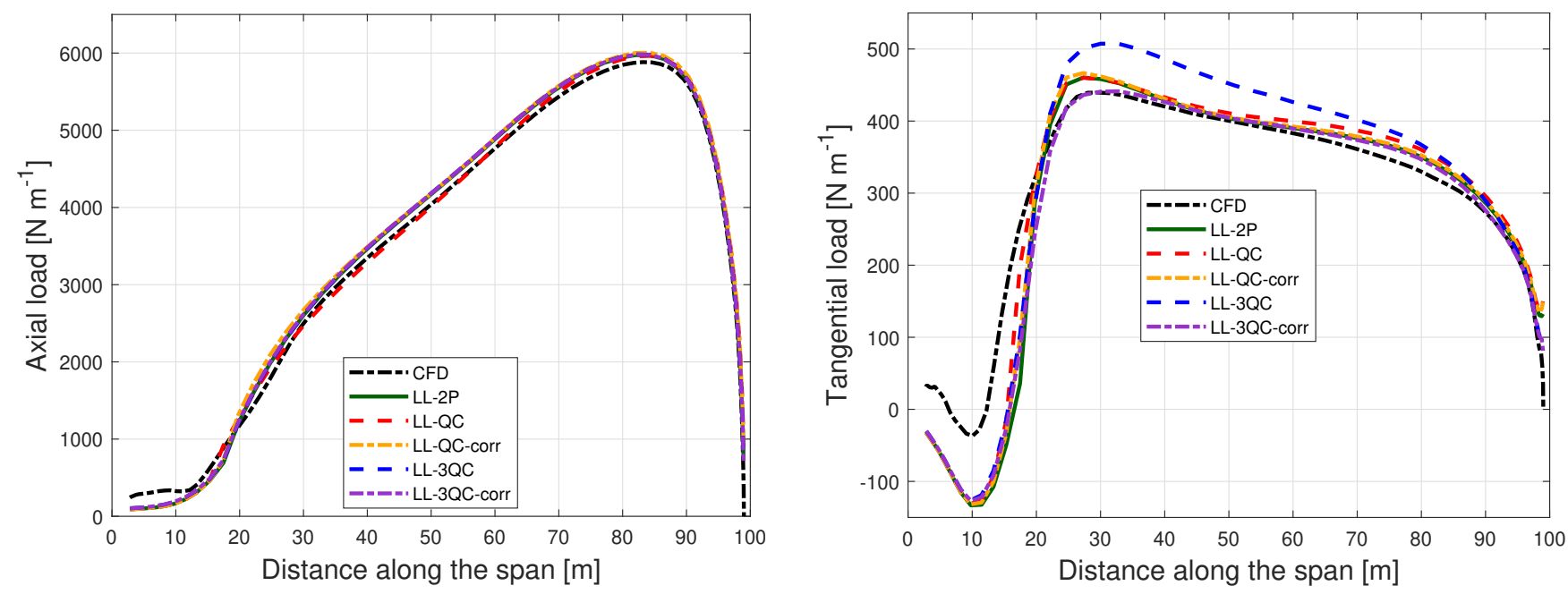

Figure 12. Comparison of axial load (left) and tangential load (right) of the straight blade with $15^{\circ}$ of downwind coning calculated from different implementations of the LL method and the fully-resolved CFD.

It can be seen from the figure that similar to the case of upwind coning, all LL methods expect LL-QC are predicting almost identical axial loads and are similar to the prediction by the fully-resolved CFD. The LL-QC method underestimates the axial load compared to other LL methods. For the tangential load, LL-3QC predicts an especially higher load compared to the predictions by other LL methods, which only show a small difference between each other and are similar to the fully-resolved CFD result. Similar as for the upwind coning case, the difference of the loads of the downwind coning straight blade with respect to the straight blade without cone calculated from different LL methods and the fully-resolved CFD are plotted in Fig. 13 for a detailed comparison. 

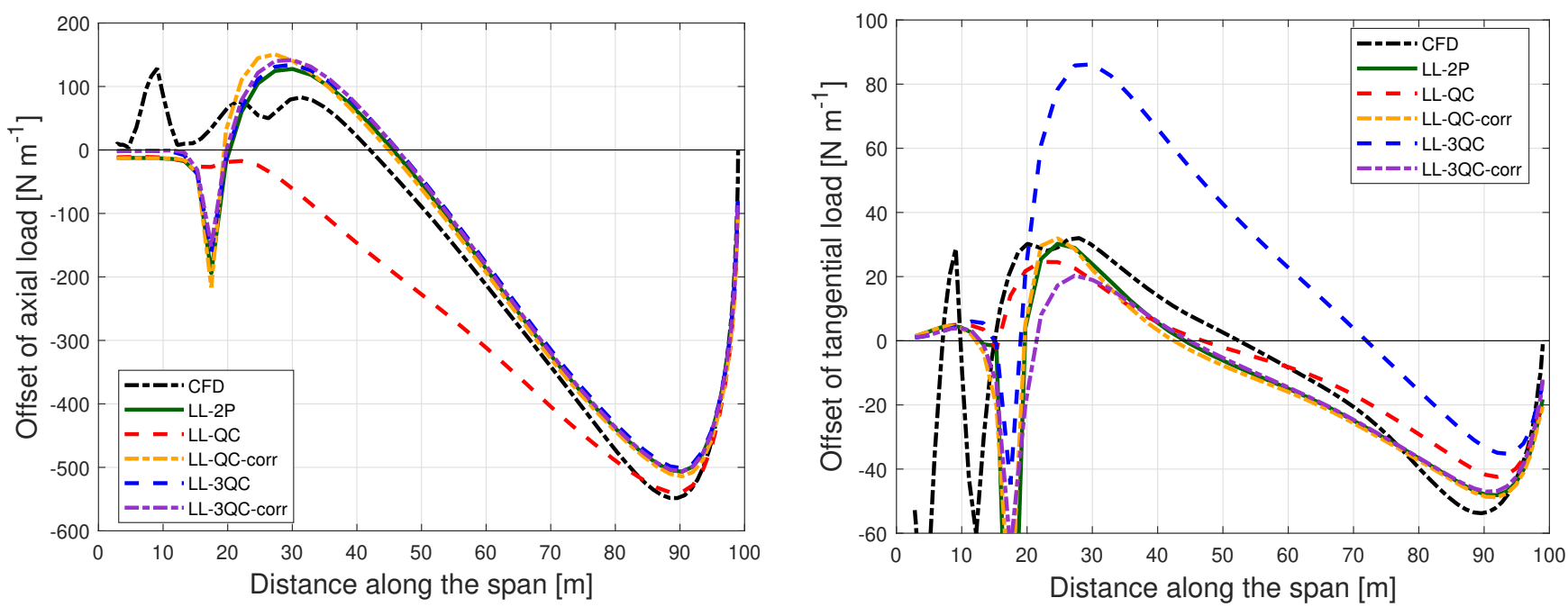

Figure 13. Comparison of the difference of the axial load (left) and tangential load (right) of the straight blade with $15^{\circ}$ of downwind coning compared to the straight blade with zero cone calculated from different implementations of the LL method and the fully-resolved CFD.

For the LL-QC method, the axial load is underestimated and shows relatively large difference compared to the load calculated using LL-2P. As has been explained for the upwind coned case, the reason is that the magnitude of the lift is not correctly calculated using LL-QC. On the other hand, the tangential load calculated from LL-QC is slightly overestimated compared to the LL-2P method but they are still in reasonably good agreement. The reason is, as has been explained for the upwind coning case, the lift force is applied in the correct direction despite the magnitude of the calculated lift force having some offsets. After applying the correction, the axial load from LL-QC-corr is now in good agreement with LL-2P. The tangential load predicted by LL-QC-corr is also in improved agreement with LL-2P.

For the LL-3QC method, the axial load is in good agreement with LL-2P since the magnitude of the lift is correctly modeled. However, LL-3QC especially overestimates the tangential load compared to LL-2P. As has been explained for the upwind coning case, this is because the lift force is not applied to the correct direction and result in an additional effective drag force. After applying the correction, the tangential load calculated from LL-3QC-corr is in significantly improved agreement with LL-2P.

In summary, all of the corrected methods are having consistently good performance for either upwind or downwind dihedral cases. The performance of LL-QC-corr and LL-3QC-corr are almost identical to LL-2P, and all of them are categorized as the full model since they align with the conclusions from unsteady 2-D airfoil theory. The important aspect is to use a correction such that the modeling effectively mimics the behaviors according to the thin airfoil theory.

\subsection{BEM method}

The importance of consistently using the 2-D airfoil data when using the BEM method to calculate the loads of the dihedral blades is tested in this section. Again, we focus on the special case of a pure dihedral blade without sweep under steady- 
state operating conditions. The blades for the test are the straight blade with $15^{\circ}$ of upwind coning and $15^{\circ}$ of downwind coning. In addition, the results of the straight blade without cone are also included as the reference. The axial and tangential loads calculated from BEM with and without the correction (by enabling or disabling the dynamic stall model) are labeled as BEM-corr and BEM, and are compared in Fig. 14.
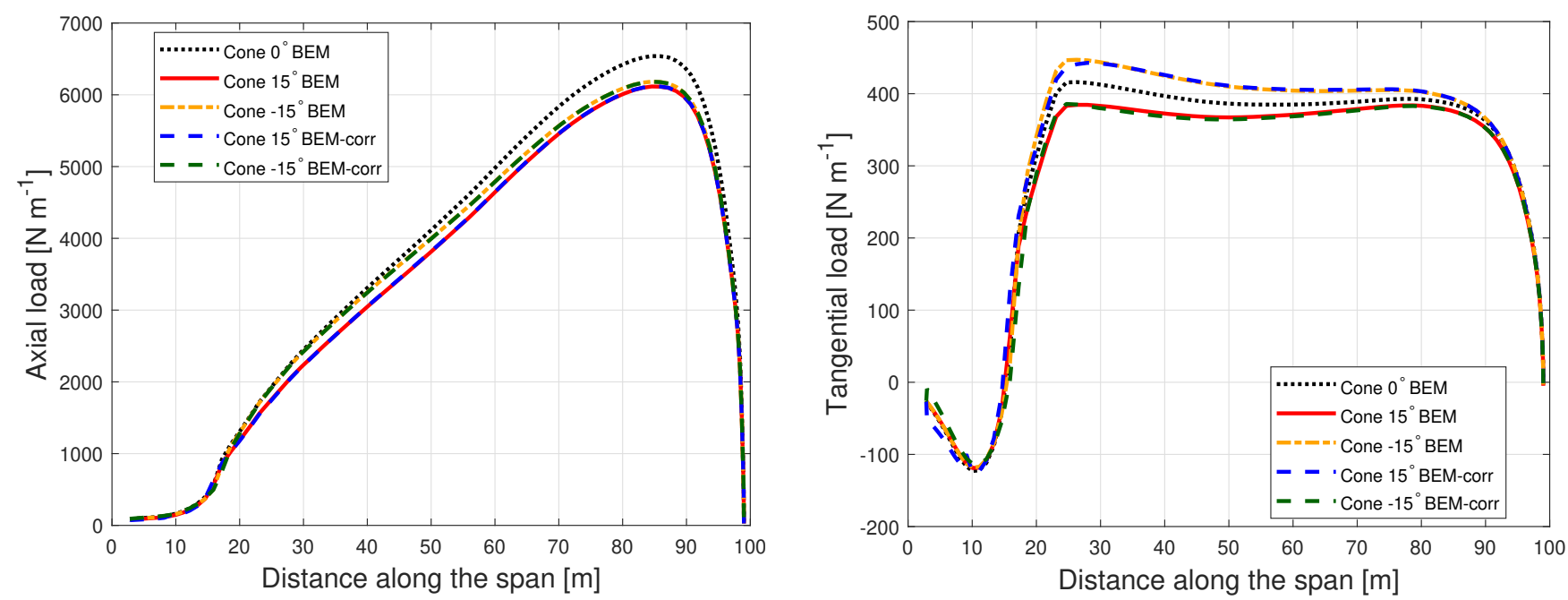

Figure 14. Comparison of axial load (left) and tangential load (right) of the straight blade without cone, with $15^{\circ}$ of upwind coning and with $15^{\circ}$ of downwind coning (labeled as Cone $-15^{\circ}$ ) calculated from the BEM method with and without the one-point lifting-line correction (labeled as BEM-corr and BEM). The results of the straight blade without cone is also included for reference.

The axial load of the upwind coned blade and the downwind coned blade are having small differences, and are lower compared to the blade without cone. For either upwind or downwind coned blade, the axial loads show only a negligible difference with and without the correction. For the tangential load of the coned blades, when including the one-point correction, the results show a relatively large difference compared to using only the quasi-steady aerodynamics. This means when using the BEM method that only uses three-quarter-chord information and without the correction for a dihedral blade operating at steady-state conditions under uniform inflow, if directly using the quasi-steady aerodynamics, the thrust force is correctly calculated, but there will be a visible error for the predicted aerodynamic power. This conclusion can be generalized into unsteady cases as well. As a result, we recommend the use of the BEM module with the unsteady 2-D airfoil model ${ }^{7}$ enabled, $^{2}$ even for steady-state calculations. In the unsteady 2-D airfoil model, the generalized one-point lifting-line correction described in Sect. 4.3 should be included.

A peculiar phenomenon that can be seen in Fig. 14 is that the tangential load of the upwind coned blade with the correction is very similar to the tangential load of the downwind coned blade without the correction. Analogously, the tangential load of the upwind coned blade without the correction is very similar to the tangential load of the downwind coned blade with the

\footnotetext{
${ }^{7}$ Such as the Beddoes-Leishman type dynamic stall model in HAWC2.
} 
correction. This shows that the BEM method is not able to correctly model the influence of blade dihedral on the 3-D wake and consequently on the aerodynamic loads.

\subsection{BEVC method}

The importance of the one-point lifting-line correction when using the BEVC method to calculate the loads of the dihedral blades is shown in this section. The blades for the test are also the straight blade with $15^{\circ}$ of upwind coning, $15^{\circ}$ of downwind coning as well as the straight blade without cone. The results from the two-point approach of the LL method (LL-2P), which have shown to be in good agreement with the high-fidelity fully-resolved CFD for these test cases in Sect. 7.3, are used for the comparison. The BEM results with the one-point correction enabled are also included in the comparison to highlight the performance of the BEVC model. The BEVC method with and without the one-point correction (by enabling or disabling the dynamic stall model) are labeled as BEVC-corr and BEVC. For the $15^{\circ}$ of upwind coning case, the difference of the axial and tangential loads compared to the straight blade without cone from different methods is shown in Fig. 15.
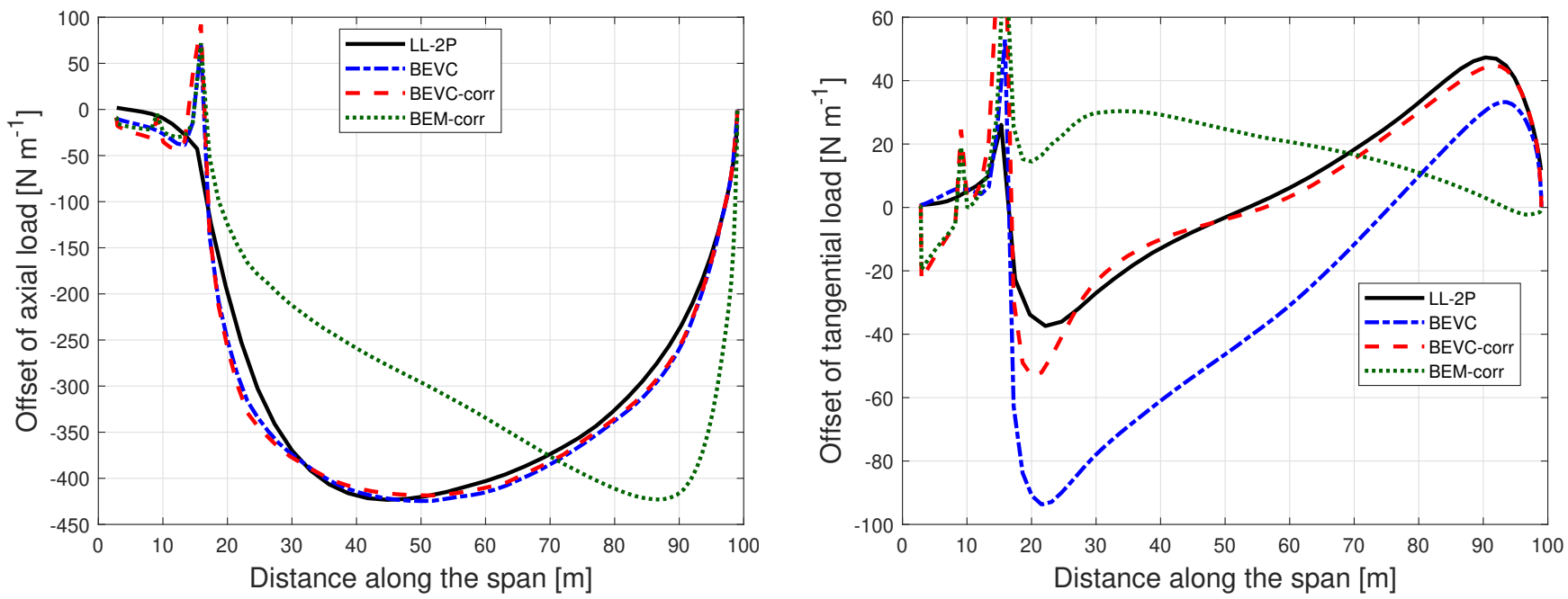

Figure 15. Comparison of the difference of axial load (left) and tangential load (right) of the straight blade with $15^{\circ}$ of upwind coning with respect to the straight blade without cone calculated from LL-2P, BEVC, BEVC with the correction (BEVC-corr) and the BEM method with the correction (BEM-corr).

For the axial load, the BEVC results with and without the correction are almost identical. This conclusion is the same as for the LL-3QC and the BEM. The difference of axial loads predicted from BEVC and BEVC-corr are in good agreement with the LL-2P. For the tangential load, BEVC-corr predicts very similar results as the LL-2P. However, if the one-point correction is not included, the BEVC method underestimates the tangential loads compared to the predictions by LL-2P. In comparison, BEMcorr predicts relatively large difference compared to LL-2P for both axial and tangential loads. This is as expected, because the BEM method, even with the one-point correction, is not able to correctly predict the influence of blade out-of-plane geometry on the loads. 
For the $15^{\circ}$ downwind coning case, the difference of the axial and tangential loads compared to the straight blade without cone from different methods is shown in Fig. 16.
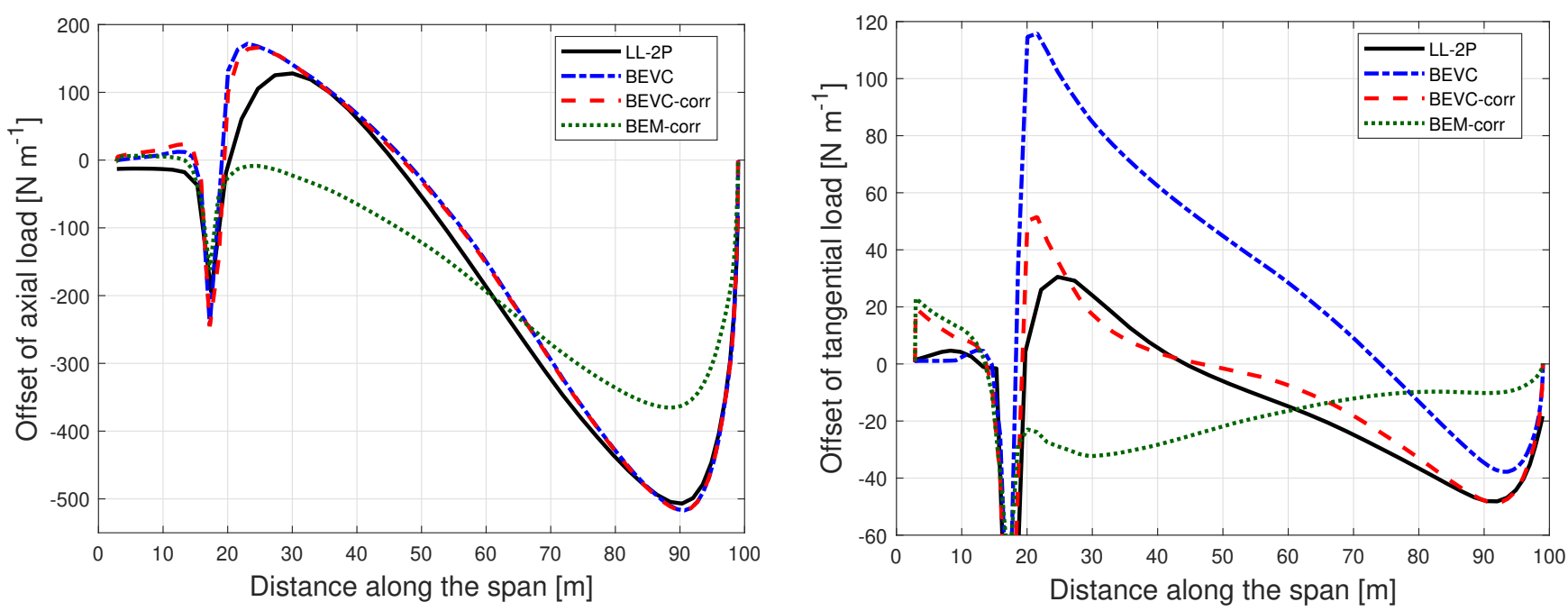

Figure 16. Comparison of the difference of axial load (left) and tangential load (right) of the straight blade with $15^{\circ}$ of downwind coning compared to the straight blade without cone calculated from LL-2P, BEVC, BEVC with the correction (BEVC-corr) and the BEM method with the correction (BEM-corr).

For the axial load, as for the upwind coned case, BEVC and BEVC-corr have almost identical results and are in good agreement with LL-2P. For the tangential load, BEVC-corr predicts similar results as the LL-2P. When directly using the quasi-steady airfoil data without the one-point correction, the BEVC method overestimates the tangential load. In comparison, BEM-corr is not able to correctly predict the influence of blade coning on the axial or tangential loads as expected.

\subsection{Actuator line method}

The actuator line (AL) used in this study is a straight line. This is equivalent to having a straight bound vortex. So, the blades used for the comparison in this section are aligned to a straight quarter-chord line instead of aligned to a straight half-chord line as in the previous sections. The axial load and tangential load of the straight blade with zero cone, with $15^{\circ}$ of upwind and downwind coning calculate from the AL method without and with the correction (labeled as AL and AL-corr) are compared with results from LL-2P. For the case of a straight blade with $15^{\circ}$ of upwind coning, the difference of the axial and tangential loads compared to the straight blade without cone is shown in Fig. 17.

It can be seen from the figure that the axial load predicted by the AL method without the correction is overestimated compared to LL-2P. This behavior is similar to LL-QC. The tangential load from the AL method is slightly overestimated. After the correction, the axial load from AL-corr is in significantly improved agreement with the result from LL-2P. For the tangential load, the shape of the result from AL-corr is in improved agreement with the result from LL-2P. However, for both axial and tangential loads, the results predicted by the AL-corr method are slightly overestimated compared to LL-2P. This 

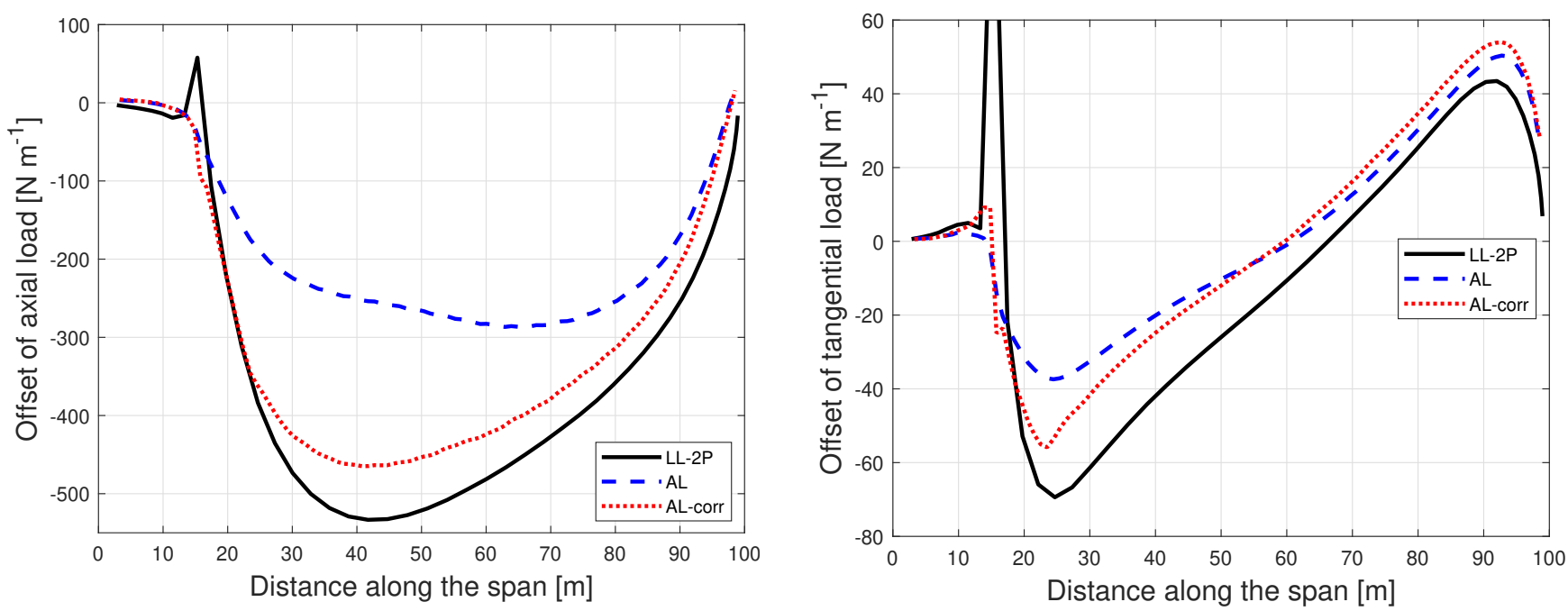

Figure 17. Comparison of the difference of axial load (left) and tangential load (right) of the straight blade with $15^{\circ}$ of upwind coning compared to the straight blade without cone calculated from LL-2P, AL without the one-point correction (AL) and AL with the one-point correction (AL-corr).

could be related to the smearing correction in the AL method that assumes the calculation point and the trailing point are both in the rotor plane (Pirrung et al., 2016), instead of following the actual blade dihedral geometry.

For the case of $15^{\circ}$ of downwind coning, the difference of the axial and tangential loads compared to the straight blade without cone is shown in Fig. 18.
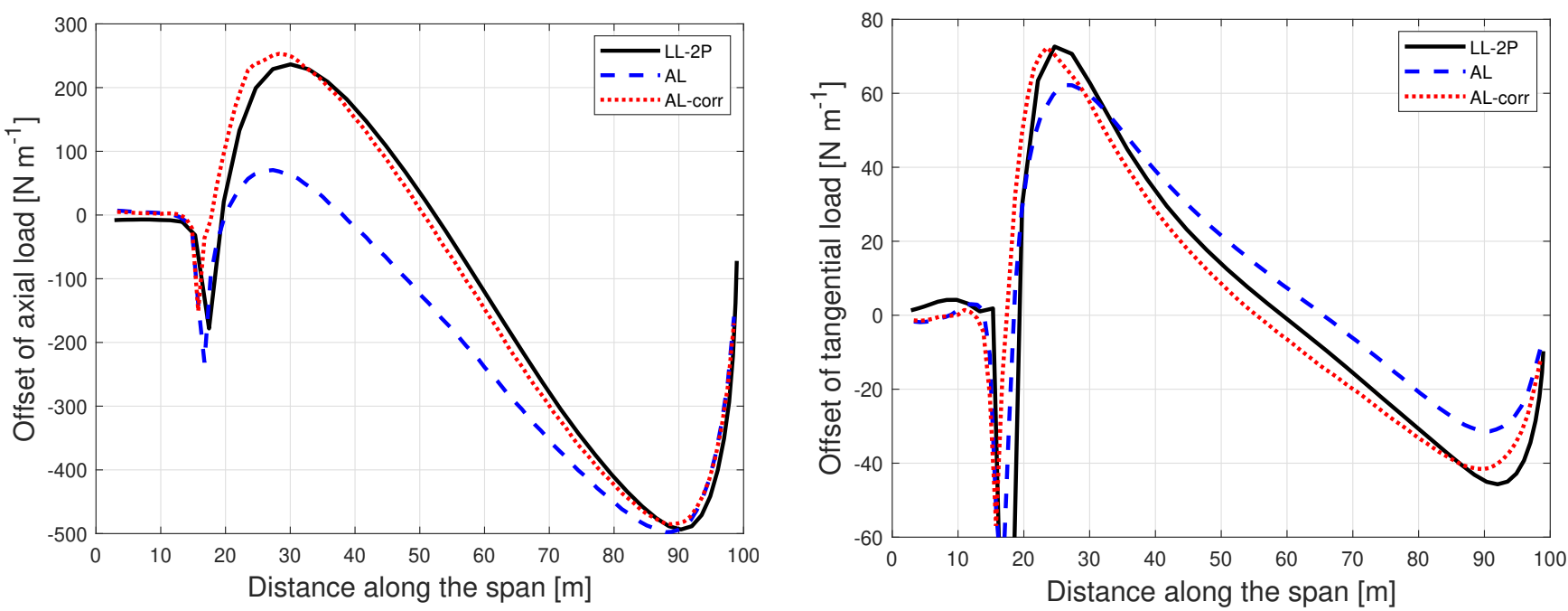

Figure 18. Comparison of the difference of axial load (left) and tangential load (right) of the straight blade with $15^{\circ}$ of downwind coning compared to the straight blade without cone calculated from LL-2P, AL without the one-point correction (AL) and AL with the one-point correction (AL-corr). 
The AL method without the correction (AL) underestimates the axial load and the behavior is similar to LL-QC. The tangential load predicted by AL is slightly overestimated compared to LL-2P. After including the one-point correction, the results from AL-corr are in improved agreement with results from LL-2P, for both axial and tangential loads.

\subsection{Integrated aerodynamic loads}

In addition to the distributed loads that are compared in Sect. 7.3 to 7.6, the importance of the one-point correction on the prediction of the integrated aerodynamic loads (thrust and power) is investigated in this section. The aerodynamic thrust and power in the present work are with the following simplified definition, where the forces are assumed to be applied at the main-axis and the contribution of airfoil moment (calculated from $C_{m}$ ) to power is neglected.

$$
\begin{aligned}
& T=N_{B} \int_{0}^{R} F_{a} \mathrm{~d} r \\
& P:=N_{B} \int_{0}^{R} r F_{t} \mathrm{~d} r
\end{aligned}
$$

where the axial force $F_{a}$ and the tangential force $F_{t}$ are with the definition of force per unit radius.

The thrust and power of the rotor with unconed straight blade as well as the upwind and downwind coned straight blades calculated from different aerodynamic models are compared. To better show the influence, the relative difference of thrust and power of the coned rotor with respect to the planar rotor with straight blades are defined as follows:

$$
\begin{gathered}
\varepsilon_{T}=\frac{T_{\text {cone }}-T_{\text {str }}}{T_{\text {str }}} \times 100 \% \\
\varepsilon_{P}=\frac{P_{\text {cone }}-P_{\text {str }}}{P_{\text {str }}} \times 100 \%
\end{gathered}
$$

The results from different models are summarized in Fig. 19 for $15^{\circ}$ upwind coned case and in Fig. 20 for $15^{\circ}$ downwind coned case.

For all the one-point approach of the generalized lifting-line methods in this comparison, if the one-point correction is applied, the predicted thrust and power of non-planar rotors have reasonably good agreement with the two-point LL method (LL-2P) and the fully-resolved CFD. This conclusion also applies to the BEM method. However, if the one-point correction is excluded and the quasi-steady polars are used directly, the results will have significant errors in either the predicted thrust or power or both, depending on the choice of the calculation point.

It can also be concluded from the results that the BEVC method does not result in significant improvement compared to the BEM method when predicting the integral thrust and power of the coned rotor with straight blades. This is because the influence of the dihedral on the distributed loads is partially canceled out when calculating the power and thrust of the whole rotor. In contrast, the improvement of BEVC over BEM when predicting thrust and power is significant when computing the rotors with curved dihedral blades as shown in the previous work (Li et al., 2021). 


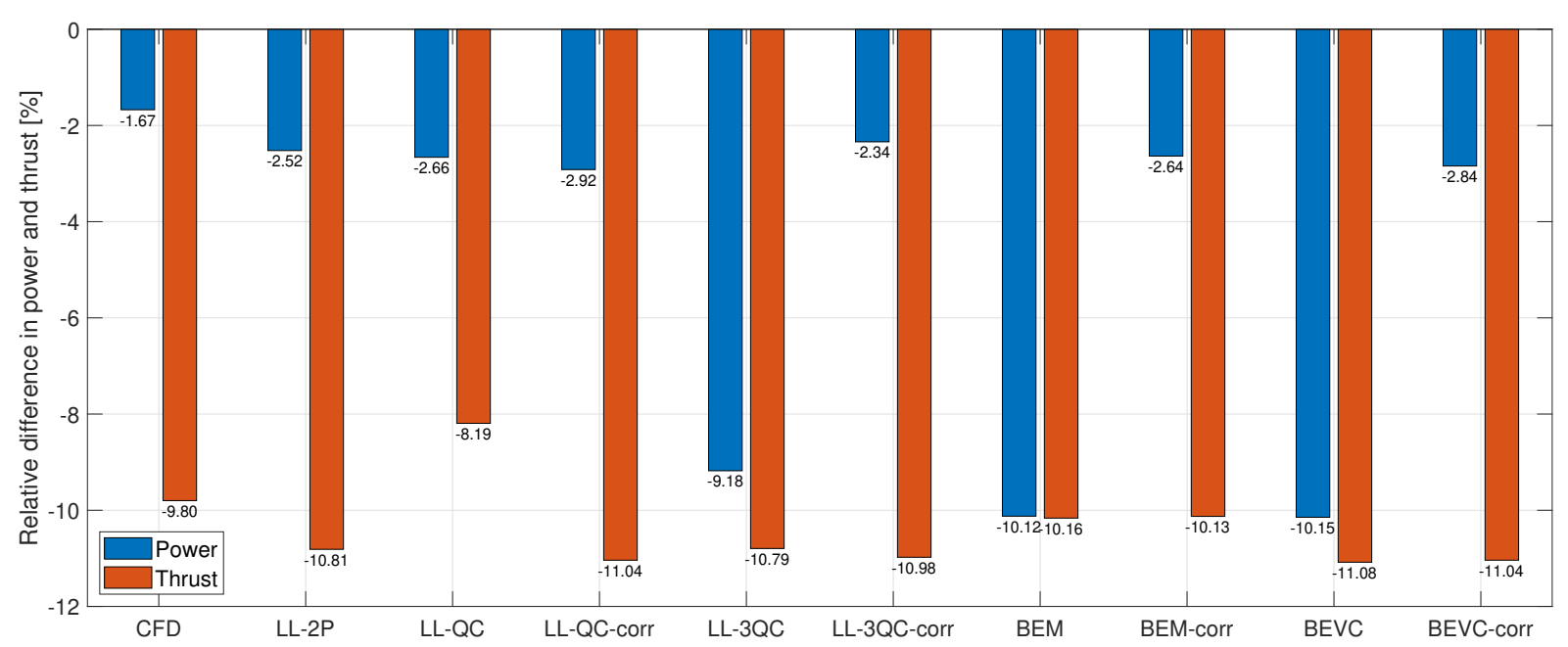

Figure 19. The relative difference of thrust and power of the rotor with straight blade with $15^{\circ}$ of upwind coning compared to the zero cone angle case.

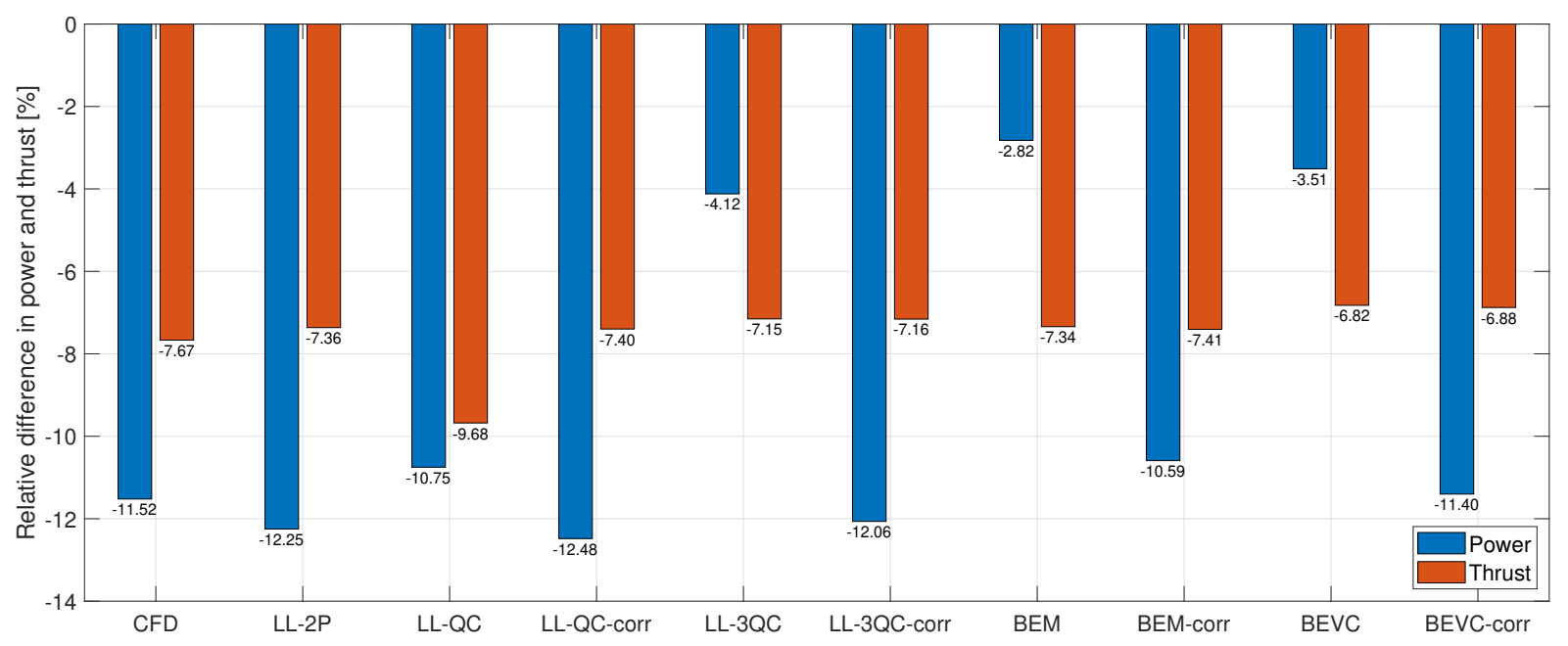

Figure 20. The relative difference of thrust and power of the rotor with straight blade with $15^{\circ}$ of downwind coning compared to the zero cone angle case. 
For the comparison of the AL method with the LL-2P method, the blades are aligned with the quarter-chord line instead of aligned with the half-chord line. The rotor thrust and power predicted by the AL method with and without the correction and also the LL-2P are summarized in Fig. 21.

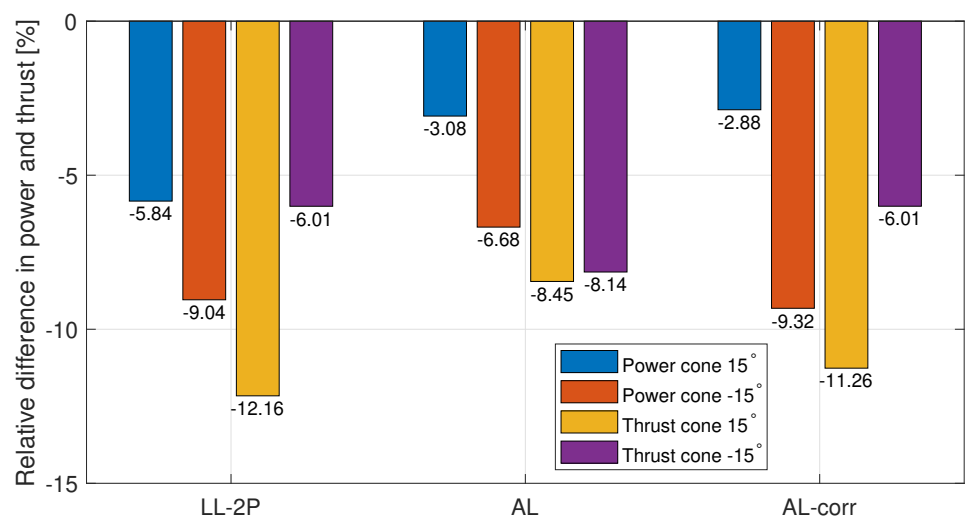

Figure 21. The relative difference of thrust and power of the rotor with $15^{\circ}$ upwind and downwind coned straight blade aligned to the quarter-chord line compared to the rotor with the same straight blade without cone predicted by the LL-2P method, the AL method without the correction (AL) and the AL method with the correction (AL-corr).

For the AL method without the one-point correction (AL), the thrust is overestimated for the upwind coned case and is underestimated for the downwind coned case. The thrust predicted by the AL method with the correction (AL-corr) is in better agreement with LL-2P for both upwind and downwind coned cases. The power predicted by AL is overestimated compared to LL-2P for both upwind and downwind coned cases. After the correction, the power of the downwind coned case is in improved agreement with LL-2P but is not improved for the upwind coned case. As has been described in Sect. 7.6, this could be related to the current smearing correction method in the AL model and future work is needed.

It is worth mentioning that the power and thrust of the cases with coned straight blades that are aligned to a straight half-chord line in Fig. 19 and 20 are having relatively large differences from the cases with the straight blades with the same cone angle but aligned to a straight quarter-chord line as in Fig. 21. The difference is approximately $1.3 \%$ in thrust and approximately $3.3 \%$ in power, for both upwind and downwind cases. For the zero cone angle cases, the results of both blades are almost identical. For the coned cases, the blades with the two different methods of blade alignment are operating at different angle of attacks. For example, at the spanwise location of $40 \mathrm{~m}$, the angle of attack of the quarter-chord aligned blade is $0.2^{\circ}$ smaller compared to the half-chord aligned blade for the $15^{\circ}$ upwind coned case and is $0.2^{\circ}$ larger for the $15^{\circ}$ downwind coned case.

The numerical tests in this section are performed on the straight blades with $15^{\circ}$ of upwind and downwind coning, which have significantly non-planar shapes. For the same straight blade at the same operating condition but with a smaller cone angle, the offset of power and thrust predicted using different generalized one-point lifting-line methods can be approximated from the results in Fig. 19 and 20. Because for the special condition that the blade has only out-of-plane dihedral and no sweep, the 
pitch rate of the effective airfoil pitching motion $\dot{\theta}$ is proportional to the cone angle $\kappa$ as shown in Eq. (28), with the small angle approximation. And for the blade sections that are operating at the linear region, the offset of lift and drag and consequently on the axial and tangential loads are then also approximately linearly proportional to the cone angle. For example, for the same straight blade that is aligned to the half-chord line and has $5^{\circ}$ of upwind coning, the relative error in power predicted by LL-3QC is inferred to be approximately $2 \%$.

\section{Conclusions and future work}

The present work describes a method where the use of key results from 2-D unsteady thin airfoil results in improved performance of generalized lifting-line methods for non-planar rotors. The conclusions from the unsteady 2-D thin airfoil theory, which are that the magnitude of the quasi-steady lift should be determined by the flow at the three-quarter-chord point and the direction of the quasi-steady lift should be determined by the flow at the quarter chord point, are highlighted. The impact of the simplification that using only one calculation point and directly using quasi-steady 2-D airfoil data as usually implemented in generalized lifting-line models is investigated. The generalized correction for such a one-point approach is given. The numerical results from the lifting-line (LL) method, the actuator line (AL) method, the blade element momentum (BEM) method and the blade element vortex cylinder (BEVC) method are compared with and without the correction. The results from fully-resolved RANS are also included as reference. The results show a large offset on the prediction of the aerodynamic performance of non-planar rotors when only using the quasi-steady aerodynamics and excluding the one-point corrections. The one-point approaches with the correction are in significantly better agreement with high-fidelity CFD results than without the corrections, for both the distributed loads and the rotor thrust and power. It is noted that the effect of the corrections is modeled correctly by a consistently implemented 2-D dynamic model, such as the Beddoes-Leishman type model in HAWC2. For this reason, it is suggested to keep such models active also for the simulation of steady-state HAWT rotors with dihedral blades.

There are several future works that are of great interest. Firstly, the comparison of the prediction of the unsteady loads using the two-point approach and the one-point approach of the lifting-line method is interesting. Secondly, the impact of correctly determining the magnitude and direction of the lift force for the curved blade with both sweep and dihedral should be investigated. Thirdly, future work on the actuator line model is necessary for the model to correctly predict the loads of blades with complex shapes. An updated smearing correction for actuator lines with curved shapes is an example.

\section{Appendix A: Nomenclature}

$a$ non-dimensioned pitching axis location

a centrifugal acceleration vector

$b$ half chord length

$730 c$ chord length

$C(k)$ Theodorsen lift deficiency function 
https://doi.org/10.5194/wes-2021-163

Preprint. Discussion started: 7 January 2022

(c) Author(s) 2022. CC BY 4.0 License.

$C_{L} \quad$ lift coefficient

$C_{D} \quad$ drag coefficient

$C_{m}$ moment coefficient

$D$ drag force

$F_{a} \quad$ axial force, with the definition of force per unit length radius

$F_{t}$ tangential force, with the definition of force per unit length radius

$F_{\text {tot }} \quad$ 2-D total force

$k$ reduced frequency

$740 \quad L \quad$ lift force

$N \quad$ 2-D normal force

$N_{B} \quad$ number of blades

$p$ position vector of the calculation point

$P \quad$ aerodynamic power of the rotor

$745 R$ radius of the rotor

$T$ 2-D tangential force, aerodynamic thrust of the rotor

$\boldsymbol{T}$ transformation matrix

$U_{0} \quad$ free wind speed

$V \quad$ free stream speed

$750 V_{\text {rel }}$ relative velocity

$w$ upwash

$\dot{x} \quad$ streamwise motion of the airfoil

$\ddot{x} \quad$ streamwise acceleration of the airfoil

$\dot{y}$ heave motion of the airfoil

$755 \quad \ddot{y} \quad$ heave acceleration of the airfoil

\section{Greek letters}

$\alpha$ angle of attack

$\tilde{\alpha}$ approximated angle of attack

$760 \Gamma$ bound vorticity strength

$\varepsilon \quad$ relative difference

$\epsilon$ normal coordinate of the 2-D airfoil

$\theta \quad$ flow angle seen by the 2-D airfoil

$\dot{\theta}$ airfoil pitch rate

$765 \quad \ddot{\theta} \quad$ airfoil pitching acceleration

$\kappa$ dihedral angle 
https://doi.org/10.5194/wes-2021-163

Preprint. Discussion started: 7 January 2022

(c) Author(s) 2022. CC BY 4.0 License.

(c) (1)

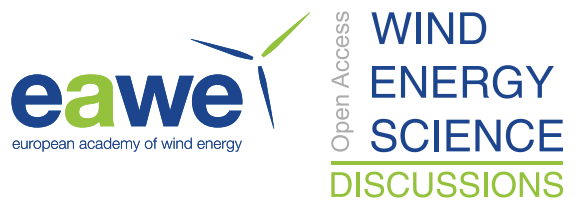

$\xi \quad$ chordwise coordinate of the 2-D airfoil

$\rho$ density of air

$\phi \quad$ sectional flow angle

$770 \Omega \quad$ rotor speed

$\Omega$ rotational velocity vector

\section{Subscripts}

$1 / 4$ at the quarter-chord point

$7753 / 4 \quad$ at the three-quarter-chord point

$a$ in the axial direction

$t$ in the tangential direction

$r$ relative value

$i \quad$ induced value

780 str straight blade

cone coned blade

heav due to heaving motion

stream due to streamwise acceleration

acc due to acceleration

pitchrate due to pitch rate

\section{Superscripts}

$\boldsymbol{R}$ in rotor coordinate system

$\boldsymbol{A}$ in airfoil coordinate system

$C$ circulatory part

$N C$ non-circulatory part

$Q S \quad$ quasi-steady

Author contributions. AL conducted the study as part of his $\mathrm{PhD}$ research. The present work is based on the previous work on the VAWT by MG and GRP. The unsteady 2-D thin airfoil aerodynamics is described by MG with contribution from AL. The non-circulatory force for the pure dihedral case is described by AL with contribution from MG and GRP. The one-point lifting-line correction is described by AL with contribution from MG and GRP. The implementation of the one-point correction in the lifting-line code is performed by AL. The fully-resolved CFD method is introduced by SGH, and its results are computed by SGH. The post-processing of the fully-resolved CFD results is performed by SGH with contribution from AL. The lifting-line results, BEM results and BEVC results are computed by AL and the 
https://doi.org/10.5194/wes-2021-163

Preprint. Discussion started: 7 January 2022

(C) Author(s) 2022. CC BY 4.0 License.

(c) (i)

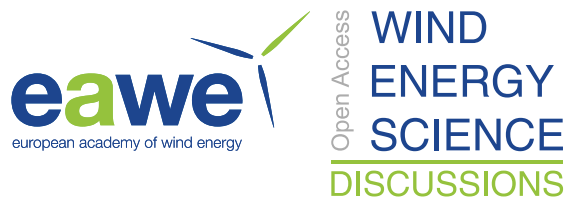

800 post-processing of the results are performed by AL. The actuator line method is described by AFM and the actuator line results are computed by AFM. All authors jointly draw the conclusions of the work and contribute to writing this work.

Competing interests. DTU Wind Energy develops and distributes HAWC2 and EllipSys3D on commercial and academic terms.

Acknowledgements. The authors would like to thank our colleague Néstor Ramos García for the help and suggestions in the lifting-line simulation using the MIRAS code that mainly developed by him. The authors would like to thank our colleague Fanzhong Meng for his contribution to the discovery of the disagreement between the aerodynamic load calculated from HAWC2 code version 12.8 and HAWCStab2 code version 2.15 for a wind turbine with large cone angle.

This work has been supported by the Smart Tip project, funded by Innovation Fund Denmark (J.nr. 7046-00023B). 


\section{References}

Bergami, L. and Gaunaa, M.: ATEFlap Aerodynamic Model, a dynamic stall model including the effects of trailing edge flap deflection,

Danmarks Tekniske Universitet, Risø Nationallaboratoriet for Bæredygtig Energi, 2012.

Bortolotti, P., Tarrés, H. C., Dykes, K., Merz, K., Sethuraman, L., Verelst, D., and Zahle, F.: Systems Engineering in Wind Energy - WP2.1 Reference Wind Turbines, Tech. rep., National Renewable Energy Laboratory (NREL), https://www.osti.gov/biblio/ 1529216-iea-wind-tcp-task-systems-engineering-wind-energy-wp2-reference-wind-turbines, 2019.

Gaunaa, M.: Unsteady two-dimensional potential-flow model for thin variable geometry airfoils, Wind Energy, 13, 167-192, https://doi.org/10.1002/we.377, 2010.

Hansen, M. H., Gaunaa, M., and Madsen, H. A.: A Beddoes-Leishman type dynamic stall model in state-space and indicial formulations, Ris $\emptyset$-R-1354, Roskilde, Denmark, 2004.

Johnson, W.: Rotorcraft aeromechanics, Cambridge University Press, 2013.

Larsen, T. and Hansen, A.: How 2 HAWC2, the user's manual, no. 1597(ver. 3-1)(EN) in Denmark. Forskningscenter Ris $\varnothing$. Ris $\varnothing-R$, Ris $\varnothing$ National Laboratory, 2007.

Li, A., Gaunaa, M., Pirrung, G. R., Ramos-García, N., and Horcas, S. G.: The influence of the bound vortex on the aerodynamics of curved wind turbine blades, Journal of Physics: Conference Series, 1618, 052 038, https://doi.org/10.1088/1742-6596/1618/5/052038, 2020.

Li, A., Gaunaa, M., Pirrung, G. R., and Horcas, S. G.: A computationally efficient engineering aerodynamic model for non-planar wind turbine rotors, Wind Energy Science Discussions, 2021, 1-44, https://doi.org/10.5194/wes-2021-100, 2021.

Loth, E., Steele, A., Ichter, B., Selig, M., and Moriarty, P.: Segmented ultralight pre-aligned rotor for extreme-scale wind turbines, in: 50th AIAA Aerospace Sciences Meeting including the New Horizons Forum and Aerospace Exposition, p. 1290, 2012.

Madsen, H. A., Larsen, T. J., Pirrung, G. R., Li, A., and Zahle, F.: Implementation of the blade element momentum model on a polar grid and its aeroelastic load impact, Wind Energy Science, 5, 1-27, https://doi.org/10.5194/wes-5-1-2020, 2020a.

Madsen, H. A., Zahle, F., Meng, F., Barlas, T., Rasmussen, F., and Rudolf, R. T.: Initial performance and load analysis of the LowWind turbine in comparison with a conventional turbine, Journal of Physics: Conference Series, 1618, 032 011, https://doi.org/10.1088/17426596/1618/3/032011, 2020b.

Martínez-Tossas, L. A. and Meneveau, C.: Filtered lifting line theory and application to the actuator line model, Journal of Fluid Mechanics, 863, 269-292, https://doi.org/10.1017/jfm.2018.994, 2019.

Menter, F. R.: Two-equation eddy-viscosity turbulence models for engineering applications, AIAA Journal, 32, $1598-1605$, 1994.

Meyer Forsting, A., Troldborg, N., Bechmann, A., and Réthoré, P.-E.: Modelling Wind Turbine Inflow: The Induction Zone, Ph.D. thesis, DTU Wind Energy, Denmark, https://doi.org/10.11581/DTU:00000022, 2017.

Meyer Forsting, A. R., Pirrung, G. R., and Ramos-García, N.: A vortex-based tip/smearing correction for the actuator line, Wind Energy Science, 4, 369-383, https://doi.org/10.5194/wes-4-369-2019, 2019a.

Meyer Forsting, A. R., Pirrung, G. R., and Ramos-García, N.: Actuator-Line-Smearing-Correction, https://doi.org/10.11583/DTU.9752285.v1, 2019b.

Meyer Forsting, A. R., Pirrung, G., and Ramos García, N.: Brief communication: A fast vortex-based smearing correction for the actuator line, Wind Energy Science, https://doi.org/10.5194/wes-2019-67, 2020.

Michelsen, J. A.: Basis3D - a Platform for Development of Multiblock PDE Solvers, Tech. Rep. AFM 92-05, Technical University of Denmark, 1992. 
https://doi.org/10.5194/wes-2021-163

Preprint. Discussion started: 7 January 2022

(c) Author(s) 2022. CC BY 4.0 License.

(c) (1)

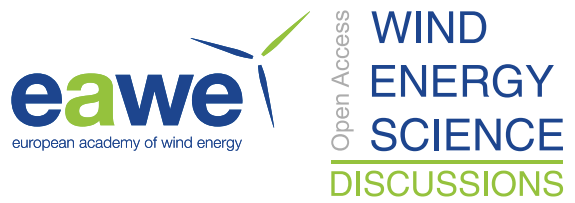

845 Michelsen, J. A.: Block structured Multigrid solution of 2D and 3D elliptic PDE's, Tech. Rep. AFM 94-06, Technical University of Denmark, 1994.

Phillips, W. F. and Snyder, D. O.: Modern adaptation of Prandtl's classic lifting-line theory, Journal of Aircraft, 37, 662-670, 2000.

Pirrung, G., Madsen, H. A., Kim, T., and Heinz, J.: A coupled near and far wake model for wind turbine aerodynamics, Wind Energy, https://doi.org/10.1002/we.1969, 2016.

Pirrung, G. R. and Gaunaa, M.: Dynamic stall model modifications to improve the modeling of vertical axis wind turbines, DTU Wind Energy E-0171, Roskilde, Denmark, 2018.

Ramos-García, N., Sørensen, J., and Shen, W.: Three-dimensional viscous-inviscid coupling method for wind turbine computations, Wind Energy, 19, 67-93, 2016.

Schepers, J., Boorsma, K., Madsen, H., Pirrung, G., et al.: IEA Wind TCP Task 29, Phase IV: Detailed Aerodynamics of Wind Turbines, IEA Wind, https://doi.org/10.5281/zenodo.4817875, 2021.

Sørensen, N. N.: General Purpose Flow Solver Applied to Flow over Hills, Ris $\varnothing-R-827-(E N)$, Risø National Laboratory, Roskilde, Denmark, 1995.

Sørensen, N. N.: HypGrid2D a 2-D Mesh Generator, Risø-R- 1035-(EN), Risø National Laboratory, Roskilde, Denmark, 1998.

Sørensen, J. N. and Shen, W. Z.: Numerical Modeling of Wind Turbine Wakes, Journal of Fluids Engineering, 124, 393-399, https://doi.org/10.1115/1.1471361, 2002.

Theodorsen, T.: General theory of aerodynamic instability and the mechanism of flutter, Tech. Rep. NACA No. 496, National Advisory Committee for Aeronautics, 1935.

Troldborg, N., Sørensen, J., and Mikkelsen, R.: Actuator Line Modeling of Wind Turbine Wakes, Ph.D. thesis, Technical University of Denmark, 2009.

865 Van Dyke, M.: Perturbation methods in fluid mechanics, The Parabolic Press, 1975.

Zahle, F.: Parametric Geometry Library (PGL), Tech. rep., https://gitlab.windenergy.dtu.dk/frza/PGL, 2019. 